Supporting Information for

\title{
Super-resolution imaging of energy transfer by
}

\section{intensity-based STED-FRET}

Alan M. Szalai ${ }^{1}$, Bruno Siarry ${ }^{1}$, Jerónimo Lukin ${ }^{2}$, Sebastián Giusti ${ }^{2}$, Nicolás Unsain ${ }^{3,4}$, Alfredo Cáceres $^{5}$, Florian Steiner ${ }^{6}$, Philip Tinnefeld ${ }^{6}$, Damián Refojo ${ }^{2}$, Thomas M. Jovin ${ }^{7}$, Fernando D. Stefani ${ }^{1,8^{*}}$

1 Centro de Investigaciones en Bionanociencias (CIBION), Consejo Nacional de Investigaciones Científicas y Técnicas (CONICET), Godoy Cruz 2390, C1425FQD Ciudad Autónoma de Buenos Aires, Argentina

${ }^{2}$ Instituto de Investigación en Biomedicina de Buenos Aires (IBioBA)-CONICET-Partner Institute of the Max Planck Society, Godoy Cruz 2390, C1425FQD Ciudad Autónoma de Buenos Aires, Argentina

${ }^{3}$ Laboratorio de Neurobiología, Instituto de Investigación Médica Mercedes y Martín Ferreyra (INIMEC, CONICET), Universidad Nacional de Córdoba (UNC), Friuli 2434, 5016 Córdoba, Argentina.

${ }^{4}$ Cátedra de Biología Celular y Molecular, Escuela de Biología; Centro de Biología Celular y Molecular (CeBiCeM, FCEFyN - UNC), Facultad de Ciencias Exactas Físicas y Naturales, Universidad Nacional de Córdoba, Córdoba, Argentina.

5 Instituto Universitario de Ciencias Biomédicas Cordoba (IUCBC), Centro de Investigación Medicina Traslacional Severo Amuchástegui (CIMETSA) UV- INIMEC-CONICET-UNC. Córdoba. Argentina 
${ }^{6}$ Department of Chemistry and Center for NanoScience, Ludwig-Maximilians-Universität München, Butenandtstr. 5-13 Haus E, 81377 München, Germany

${ }^{7}$ Laboratory of Cellular Dynamics, Max Planck Institute for Biophysical Chemistry, am Fassberg 11, 37077 Göttingen, Germany.

${ }^{8}$ Departamento de Física, Facultad de Ciencias Exactas y Naturales, Universidad de Buenos Aires, Güiraldes 2620, C1428EHA Ciudad Autónoma de Buenos Aires, Argentina

\section{Table of Contents}

Supplementary Section 1. STED nanoscope and fluorophores..................................................... 3

Supplementary Section 2. DNA origami samples ........................................................................... 5

Supplementary Section 3. Assessment of resolution........................................................................ 7

Supplementary Section 4. Intensity-based FRET formula derivation …............................................ 8

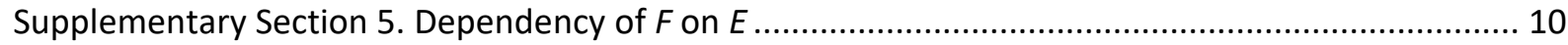

Supplementary Section 6. STED-FRET imaging and analysis ........................................................ 11

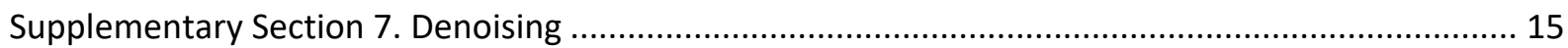

Supplementary Section 8. Evaluation of STED-FRET through simulations....................................... 17

Supplementary Section 9. F distributions from Figure 4. ............................................................. 22

Supplementary Section 10. Preparation of biological samples ........................................................ 22

Supplementary Section 11. DNA sequences used for building the DNA origami .............................. 24

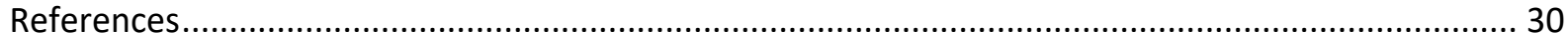




\section{Supplementary Section 1. STED nanoscope and fluorophores}

The excitation path of the home-built STED nanoscope consists of two linearly polarized pulsed lasers at $594 \mathrm{~nm}$ (100 ps pulse-width, PicoQuant LDH-D-TA-595) and $640 \mathrm{~nm}$ (200 ps pulsewidth, PicoQuant LDH-P-C-640B), both operating at $40 \mathrm{MHz}$ repetition. For each laser, light was coupled into a polarization-maintaining single-mode fiber (Thorlabs P3-488PM-FC-5) using a fiber collimator (Schäffer + Kirchhoff 60FC-4-A7.5-01). Light exiting the fiber was collimated $(\mathrm{f}=30 \mathrm{~mm})$ in order to obtain a TEM00 excitation beam and circular polarization was adjusted using a broadband (400-800 nm) quarter-wave plate (Thorlabs AQWP05M-600) and a broadband (400-800 nm) half-wave plate (Thorlabs AHWP05M-600).

For depletion, a linearly polarized pulsed laser at $775 \mathrm{~nm}$ was used (800 ps pulse-width, Onefive Katana HP), operating at $40 \mathrm{MHz}$. Light was coupled into a polarization-maintaining singlemode fiber (Thorlabs P3-630PM-FC-5) using a fiber collimator (Schäffer + Kirchhoff 60FC-4A11-02). STED light exiting the fiber was then collimated ( $\mathrm{f}=30 \mathrm{~mm}$ ) and sent through a $2 \pi$ vortex phase plate (Vortex Photonics, V-775-70). Circular polarization was adjusted using a broadband (690-1200 nm) quarter-wave plate (Thorlabs AQWP05M-980) and a broadband (690-1200 nm) half-wave plate (Thorlabs AHWP05M-980).

The three laser beams were combined using a long pass dichroic mirror for the $594 \mathrm{~nm}$ excitation beam (ZT594RDC, Chroma), a notch filter at 22 degrees for the $640 \mathrm{~nm}$ excitation beam (NF03-658-25, Semrock), and a $5 \mathrm{~mm}$ short pass dichroic mirror for the STED beam (Z780SPRDC, Chroma). Lateral beam scanning was performed by a system composed of a home-made galvanometric scanner, a scanning lens (Leica), and a tube lens (Leica). The sample was mounted on an XYZ piezoelectric nanopositioning stage (Thorlabs NanoMax MAX311D/M), and light was focused with an objective of 1.4 NA (Leica HCX PL APO 100x/1.40-0.70 Oil CS).

Two notch filters (NF03-785-25, Semrock) were used to reject STED wavelength from the detection path. After passing through this filter, fluorescence arising from the sample was split with a long pass dichroic mirror (FF649-Di01-25x36, Semrock). The reflected light passed 
through a notch filter (ZET592NF, Chroma) and a band-pass filter (FF01-623/24-25, Semrock), and was then focused on the "detector 1", an avalanche photodiode (APD, SPCM-AQR-13, PerkinElmer Optoelectronics). The light transmitted through the dichroic mirror was directed to a notch filter (ZET647NF, Chroma) and a band-pass filter (FF01-676/37-25, Semrock), and was then focused into the "detector 2" APD (SPCM-AQR-13, PerkinElmer Optoelectronics).

To maximize depletion, the depletion pulse was delayed $0.4 \mathrm{~ns}$ with respect to the excitation pulses. This optimum delay was determined in two steps. First, a coarse delay was established by confocal measurements of the emission intensity of $10 \mu \mathrm{M}$ solution of ATTO 594 (ATTO $647 \mathrm{~N})$ emission using the $592 \mathrm{~nm}(640 \mathrm{~nm})$ excitation laser and the $775 \mathrm{~nm}$ depletion laser. The two lasers were focused and spatially overlapped. The $775 \mathrm{~nm}$ pulses were shifted in time with respect to the excitation pulses while monitoring the emission. The optimum time delay was established when emission was minimum (maximum depletion). A fine adjustment was performed by checking the achieved resolution in samples of spectrin rings in the MPS of hippocampal neurons. In our case, we found 0.4 ns delay was optimum for both fluorophores. Alternatively, a middle point between the two optimums can be taken.

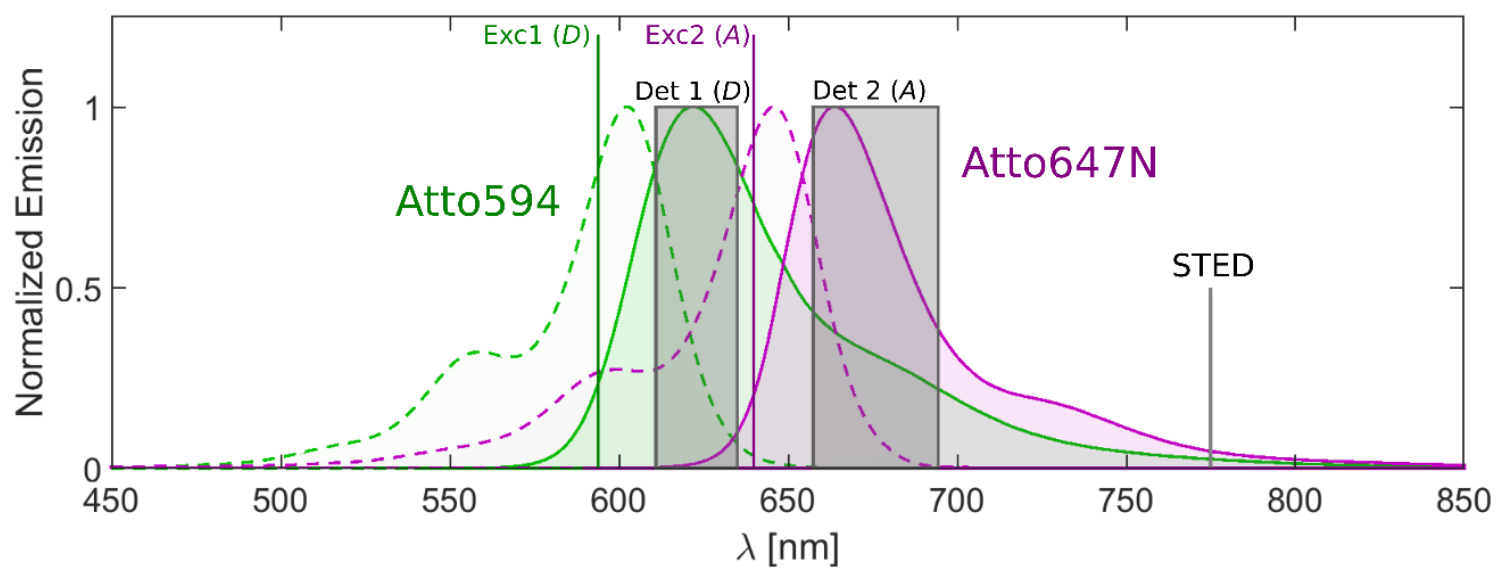

Supplementary Figure 1. Absorption and emission spectra of donor and acceptor molecules, overlapped with the band emission filters used and the wavelengths of the excitation and depletion beams. The normalized absorption spectra are shown using dotted lines, while the emission spectra are depicted with solid lines. The green color represents the donor molecule ATTO 594 and magenta plots correspond to the acceptor ATTO 647N. The excitation and depletion wavelengths are shown with vertical lines $(\operatorname{Exc1}(D)=594 \mathrm{~nm}, \operatorname{Exc} 2(A)=640 \mathrm{~nm}, \operatorname{STED}=775 \mathrm{~nm})$, and the bandpass emission 
filters used for each detection channel are depicted in rectangles (Det1 (D): from 611 to $635 \mathrm{~nm}$; Det2 (A): from 657.5 to $694.5 \mathrm{~nm})$.

Time gating of fluorescence photons was performed using a custom-made electronic board (MPI for biophysical Chemistry), setting a $9.5 \mathrm{~ns}$ detection window that started $2 \mathrm{~ns}$ after each excitation pulse to avoid detecting photons from the first ns after the excitation pulse. These photons correspond to the emission of the molecules before they could be efficiently depleted, therefore they carry information of low spatial frequency and impair the spatial resolution.

The scanning and fluorescence count acquisition were computer-controlled via an AD/DA board (National Instruments PCIe-6353) using the software ImSpector ${ }^{1}$.

Supplementary Figure 1 shows the absorption and emission spectra of the $D$ and $A$ fluorophores, the excitation and depletion laser lines, and the spectral windows used for $D$ and $A$ detection. It should be noted that $D$ and $A$ fluorophores should meet photophysical properties suitable for both FRET and STED. In this case, we selected two fluorophores that are capable of performing FRET and at the same time are suitable for STED nanoscopy using a single depletion beam at $775 \mathrm{~nm}$.

\section{Supplementary Section 2. DNA origami samples}

\section{DNA origami design}

A DNA origami was designed to test $\mathrm{i}$ ) whether STED can super-resolve FRET spots and ii) if it can do it at the single-molecule level. The DNA origami consists of a 12-helix bundle that forms a rigid nanorod as described $\mathrm{in}^{2}$, with a length of $228 \mathrm{~nm}$ and a diameter of $14 \mathrm{~nm}$. We have included two FRET spots separated by $180 \mathrm{~nm}$. Each FRET spot consists of one A (ATTO $647 \mathrm{~N}$ ) and two D (ATTO 594) molecules. The separation distance between D and A molecules was set to $7 \mathrm{~nm}$. According to the manufacturer ${ }^{3}$, the isotropic Förster radius for the ATTO 594 
- ATTO $647 \mathrm{~N}$ pair is $7.4 \mathrm{~nm}$. A separation distance of $7 \mathrm{~nm}$ ensures significant FRET while maintaining detectable donor emission.

The reason for using 2 donors and 1 acceptor molecule on each FRET spot was to guarantee a high yield of DNA-origami structures with two active FRET spots for sufficient duration to observe single-molecule anti-correlated $D$ - $A$ signals from each FRET spot. Under our experimental conditions, ATTO 594 is considerably less photostable than ATTO 647N. Although we firstly performed measurements with an origami with 1:1 $D: A$ stoichiometry, we switched to the $2 D: 1 A$ configuration because in the 1:1 case the $D$ tends to bleach early in the trace and before the $A$, and it was therefore very hard to observe traces with several anticorrelated events.

\section{DNA origami preparation}

DNA origami structures were purchased from GATTAquant $\mathrm{GmbH}$, Germany, using the sequences of ref. ${ }^{4}$ With dye modifications to ensure the labeling scheme shown in Figure $1 \mathrm{~b}$. The complete list of staple strands used in the DNA origami structures is given in Supplementary Table 1.

For folding, the scaffold strand is mixed with the staple strands (10-fold excess for unmodified staples, 100-fold excess for dye modified staples, 30-fold excess for biotin staples) in the folding buffer (1x TAE buffer consisting of $20 \mathrm{mM}$ acetic acid, $1 \mathrm{mM}$ EDTA, and $50 \mathrm{mM}$ Tris with $16 \mathrm{mM} \mathrm{MgCl}_{2}$ ). The mixture was heated to $65^{\circ} \mathrm{C}$ and slowly cooled down with a nonlinear thermal ramp over 16 hours. The excess staples were removed with polyethylene glycol (PEG) precipitation after the folding procedure. Afterwards, the samples were mixed with an equal volume of PEG precipitation buffer (1× TAE, $15 \%$ (w/v) PEG-8000, $500 \mathrm{mM} \mathrm{NaCl}, 12 \mathrm{mM}$ $\mathrm{MgCl}_{2}$ ) and centrifuged at $16 \mathrm{krcf}$ (thousand relative centrifugal force, i.e. $1000 \mathrm{~g}$ ) for $45 \mathrm{~min}$ at $4{ }^{\circ} \mathrm{C}$. After removing the supernatant, the pellet was suspended in the folding buffer as described above. Afterwards, the DNA origami structures were externally labeled with ATTO 594 and ATTO 647N modified oligonucleotides. An excess of staples (3x with respect to the extended staples) was used and incubated in a wet chamber at room temperature for $20 \mathrm{~min}$. 
The DNA origami structures were purified via gel electrophoresis. An $1.5 \%$ agarose gel containing a Tris base, acetic acid, TAE buffer $(0.5 \times$ TAE) and $12 \mathrm{mM} \mathrm{MgCl} 2$ was used at 50 $\mathrm{V}$ for 2 hours in a gel box cooled in an ice-water bath. DNA origami structures could be identified on a UV/blue illuminated table due to the numerous fluorescent dyes and recovered from the target band. The samples were stored at $-20{ }^{\circ} \mathrm{C}$ until further use.

\section{DNA origami samples}

1.5 thickness glass-bottomed chamber slides (Lab-Tek II, Thermo Fisher Scientific) were treated with $1 \mathrm{M} \mathrm{KOH}$ for $10 \mathrm{~min}$, washed (3x) with PBS, functionalized with BSA-biotin (1 $\mathrm{mg} / \mathrm{mL}, 10 \mathrm{~min}$ incubation), washed (3x) with PBS and incubated for $20 \mathrm{~min}$ with Neutravidin $(1 \mathrm{mg} / \mathrm{mL})$. Next, chambers were washed $(5 \mathrm{x})$ with TAE-12 buffer (40 mM Tris base, $20 \mathrm{mM}$ acetic acid, $1 \mathrm{mM}$ EDTA, $12 \mathrm{mM} \mathrm{MgCl} 2, \mathrm{pH}$ 8) and incubated for $30 \mathrm{~min}$ with DNA origami samples (1:10 dilution in TAE-12; final DNA origami concentration $\sim 0.1-1 \mathrm{nM})$. Next, chambers were washed (5x) with TAE-12 and filled with imaging buffer (TAE-12 for 2c and 2d-top, and a mixture of buffers $\mathrm{A}(90 \%)$ and $\mathrm{B}(10 \%)$ for $2 \mathrm{~d}$-bottom, being $\mathrm{A}=1 \mathrm{x}$ TAE, 12 $\mathrm{mM} \mathrm{MgCl} 2,2 \mathrm{mM}$ Trolox/Troloxquinone, $1 \%$ (w/v) D-(+)-Glycose; and $\mathrm{B}=1 \mathrm{mg} \mathrm{mL} \mathrm{m}^{-1}$ glucose oxidase, $0.4 \%(\mathrm{v} / \mathrm{v})$ catalase $\left(50 \mu \mathrm{g} \mathrm{mL}{ }^{-1}\right), 30 \%$ glycerol, $12.5 \mathrm{mM} \mathrm{KCl}$ in $50 \mathrm{mM}$ TRIS).

\section{Supplementary Section 3. Assessment of resolution}

The resolution of the donor and acceptor STED images were obtained using a parameter-free method based on decorrelation analysis ${ }^{5}$. Images of four origamis were aligned for these measurements. 

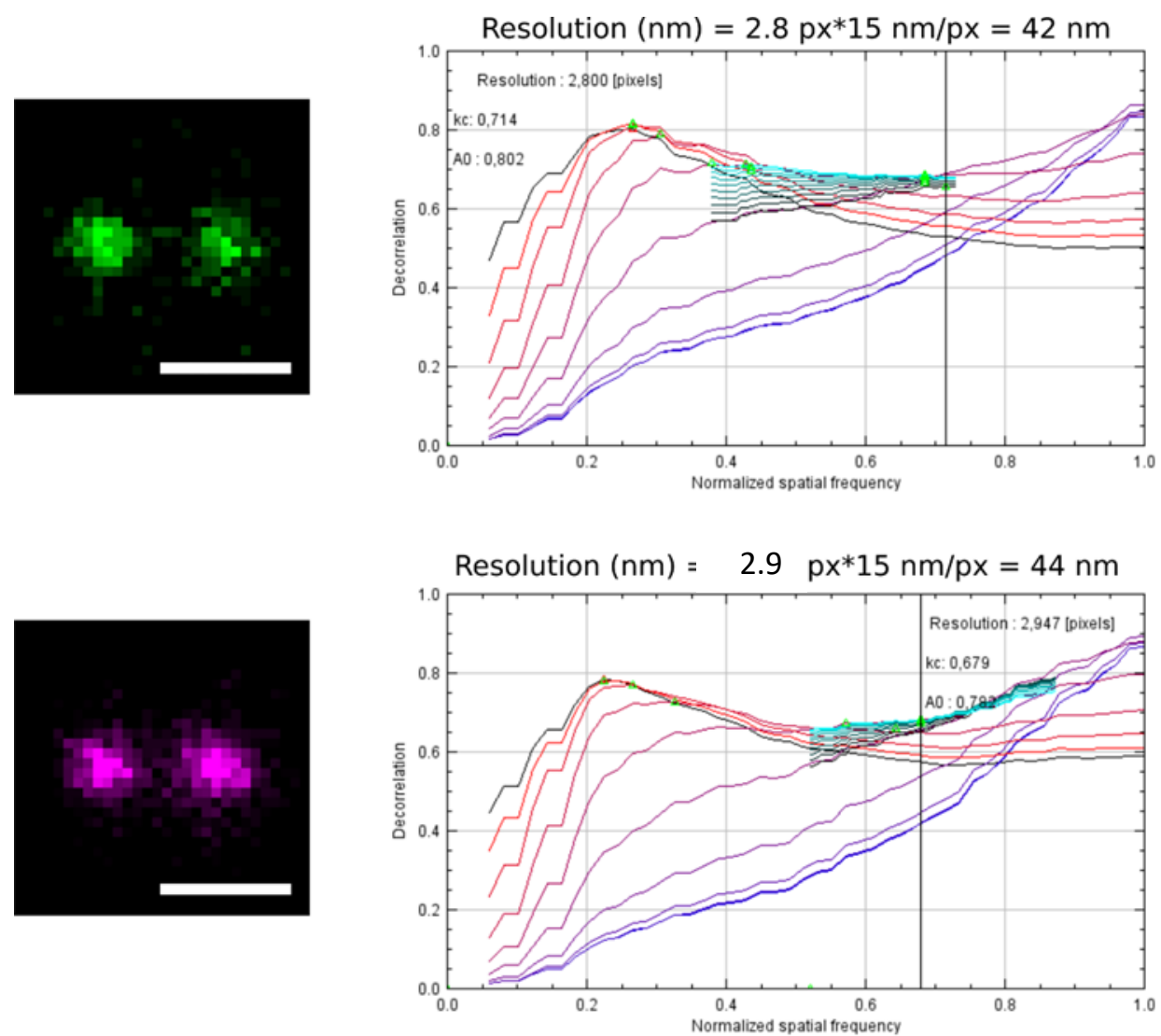

Supplementary Figure 2. Resolution measurements on STED images of DNA origami samples, for the donor and acceptor channels. Right: Output figures of the decorrelation analysis software. Decorrelation functions computed for the image-based resolution estimation for the (top) donor channel and (bottom) acceptor channel average images of the aligned DNA origami examples from Figure 1c. Left: average images of both channels. Scalebars: $200 \mathrm{~nm}$.

\section{Supplementary Section 4. Intensity-based FRET formula derivation}

The FRET transfer rate $k_{t}$ was defined in eq. (1) of the manuscript. Eq. (2) of the manuscript gives the definition of $\frac{k_{t}}{k_{f, D}}$. Here, we show how eq. (3) of the manuscript is derived using eq. (1) and (2), and the FRET and emission quantum efficiencies. 
In the presence of a depletion STED laser, the quantum efficiencies of FRET, of donor emission, and of acceptor emission are given by:

$$
\begin{aligned}
& E=\frac{k_{t}}{k_{f, D}+k_{n r, D}+k_{t}+k_{S T E D, D}} \\
& \phi_{D}=\frac{k_{f, D}}{k_{f, D}+k_{n r, D}+k_{t}+k_{S T E D, D}} \\
& \phi_{A}=\frac{k_{f, A}}{k_{f, A}+k_{n r, A}+k_{S T E D, A}}
\end{aligned}
$$

where $k_{f, D}$ and $k_{f, A}$ are the intrinsic radiative decay rates of the donor and acceptor, respectively. $k_{n r, D}$ and $k_{n r, A}$ are the intrinsic non-radiative decay rates of the donor and acceptor, respectively. $k_{S T E D, D}$ and $k_{S T E D, A}$ are the steady-state effective depletion rates of the donor and acceptor, respectively.

Under conditions of acceptor excitation, the fluorescence signal is only detected in the acceptor detection channel, and given by:

$$
F_{A}^{A}=I^{A} \sigma_{A}^{A} C_{A} \phi_{A} d_{A}^{A}
$$

where $I^{A}$ is the excitation light intensity at the acceptor excitation wavelength. $\sigma_{A}^{A}$ is the acceptor molecular absorption cross-section at the acceptor excitation wavelength; $C_{A}$ is the acceptor concentration; and $d_{A}^{A}$ is the fraction of the acceptor emission spectrum that is detected in the acceptor channel. Under conditions of donor excitation, the steady-state fluorescence signal detected in the donor detection channel takes on an analogous form:

$$
F_{D}^{D}=I^{D} \sigma_{D}^{D} C_{D} \phi_{D} d_{D}^{D}
$$

In contrast, the fluorescence signal detected in the acceptor channel under donor excitation includes three terms, direct acceptor excitation, FRET, and donor emission cross-talk:

$$
F_{A}^{D}=I^{D} \sigma_{A}^{D} C_{A} \phi_{A} d_{A}^{A}+I^{D} \sigma_{D}^{D} C_{D} E \phi_{A} d_{A}^{A}+I^{D} \sigma_{D}^{D} C_{D} \phi_{D} d_{D}^{A}
$$

From equations (S.1)-(S.6) and eq. (2), one obtains the expression shown in eq. (3) for $k_{t} / k_{f, D}$. 


\section{Supplementary Section 5. Dependency of $F$ on $E$}

$F$ values were calculated using eq. 3 of the manuscript for different scenarios. The fluorescence signals $\left(F_{A}^{D}, F_{D}^{D}\right.$ and $\left.F_{A}^{A}\right)$ were calculated for $E$ values ranging from 0 to 1 , using the photophysical parameters informed by the manufacturer for ATTO 594 and ATTO 647N, and the ratio of the donor emission detected in the acceptor and donor channels experimentally determined in our setup. In Supplementary Figure 3, we show how the $F$ vs. $E$ dependence varies when the donor or acceptor fluorescence quantum yields (in the absence of FRET) are slightly modified around their mean values.

a)
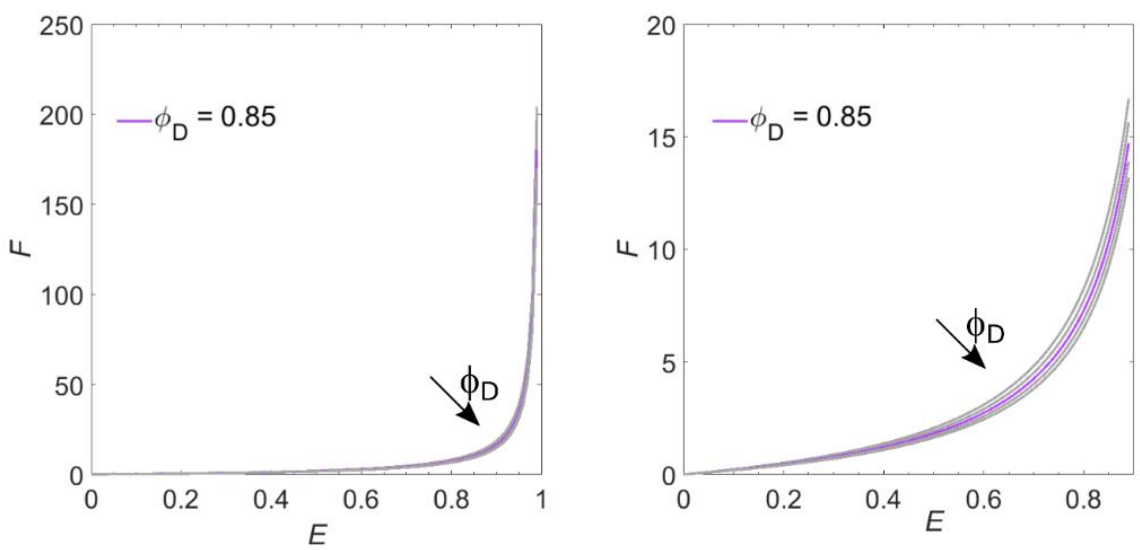

b)
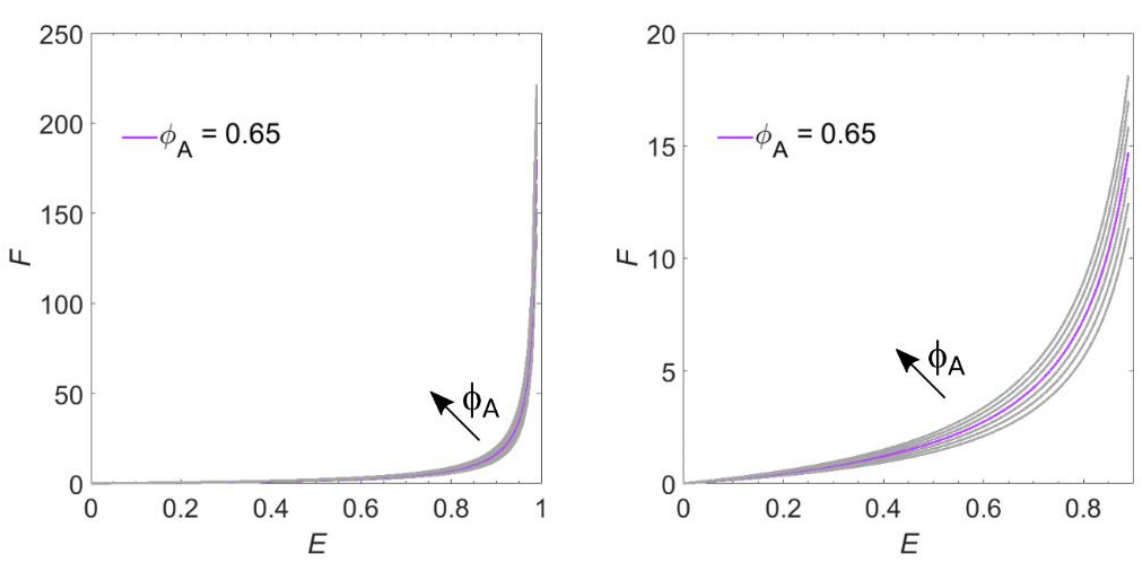

Supplementary Figure 3. $F$ dependence on $E$ under the experimental conditions used in this work. (a) $F$ dependence on $E$ when $\phi_{A}=0.65$ (fluorescence quantum yield reported for ATTO $647 \mathrm{~N}$ by the manufacturer), $\quad d_{D}^{A}=1.38 * d_{D}^{D}$, and $1.25 \sigma_{D}^{D}=3.8 \sigma_{A}^{D}=\sigma_{A}^{A}$ (relations obtained from the specifications of ATTO 594 and ATTO $647 \mathrm{~N}$ reported by the manufacturer). The different curves 
correspond to different values of $\phi_{D}$ in the absence of FRET, ranging from 0.75 to 0.95 , in 0.05 steps (the range is centred in 0.85 , the value informed by the manufacturer for ATTO 594). Left: $E$ values ranging from 0 to 1 . Right: $E$ values ranging from 0 to 0.9 . The curves corresponding to $\phi_{D}=0.85$ curve is highlighted in purple. (b) $F$ dependence on $E$ using the same parameters as in a), but in this case $\phi_{D}$ in the absence of FRET was fixed to 0.85 , and $\phi_{A}$ was varied between 0.5 and 0.8 , in 0.05 steps. Left: $E$ values ranging from 0 to 1 . Right: $E$ values ranging from 0 to 0.9 . The $\phi_{A}=0.65$ curve is coloured purple.

We note that the conversion of $F$ into $E$ shown above is only approximate. An accurate determination of $E$ is not straightforward because it depends on the precise knowledge of all parameters involved. In addition, computing $E$ accurately in the case of STED FRET requires a quantitative analysis of the influence of the depletion process on the intensity of the $D$ and $A$ molecules. This should include, if used, the effect of time-gated detection.

\section{Supplementary Section 6. STED-FRET imaging and analysis}

\section{Imaging conditions}

The images of the DNA origamis shown in Figure 1c were acquired under $594 \mathrm{~nm}$ excitation, with laser power measured at the objective pupil of $5 \mu \mathrm{W}$ (confocal image) and $15 \mu \mathrm{W}$ (STED image); these values correspond to power densities of $15 \mathrm{~kW} / \mathrm{cm}^{2}$ and $45 \mathrm{~kW} / \mathrm{cm}^{2}$, respectively. The pulse energy of the depletion laser $(775 \mathrm{~nm})$ was set to $4 \mathrm{~nJ}$. The pixel size was $40 \mathrm{~nm}$ for the confocal image, and $15 \mathrm{~nm}$ (single DNA origamis) and $20 \mathrm{~nm}$ for STED imaging (5 x 5 $\mu \mathrm{m}^{2}$ field of view). The pixel dwell time was $0.1 \mathrm{~ms}$ (confocal) and $0.5 \mathrm{~ms}$ (STED).

Confocal-FRET time traces (Figure 1d) were obtained by measuring successively 128 confocal images of $1 \mu \mathrm{m} \times 1 \mu \mathrm{m}$ (50 nm pixel size, $0.3 \mathrm{~ms}$ dwell time), under $594 \mathrm{~nm}$ excitation (5 $\mu \mathrm{W})$, and integrating the emission intensity in a $600 \mathrm{~nm}$ x $600 \mathrm{~nm}$ region centered on the origami structure. STED-FRET time traces were obtained by measuring 16 successive STED images of 
$600 \mathrm{~nm}$ x $600 \mathrm{~nm}$ (20 nm pixel size, $0.3 \mathrm{~ms}$ dwell time), using $594 \mathrm{~nm}$ as excitation wavelength $(5 \mu \mathrm{W})$ and a pulse energy of $4 \mathrm{~nJ}$ for the $775 \mathrm{~nm}$ depletion beam. Both for STED-FRET and Confocal-FRET, the cross-talk used for photon counts correction was measured independently, using DNA-origamis lacking $A$ molecules.

Confocal- and STED-FRET imaging of immunostained microtubules were performed under the following conditions: laser intensities of $0.2 \mu \mathrm{W}$ (confocal image) and $1.2 \mu \mathrm{W}$ (STED image) for $D$ excitation (594 nm); $0.2 \mu \mathrm{W}$ (confocal image) and $0.7 \mu \mathrm{W}$ (STED image) for $A$ excitation $(640 \mathrm{~nm})$, and a $775 \mathrm{~nm}$ laser pulse energy of $4 \mathrm{~nJ}$. Measurements of actin and spectrin were carried out using laser intensities of $0.5 \mu \mathrm{W}$ (confocal image) and $10 \mu \mathrm{W}$ (STED image) for $D$ excitation (594 nm); $0.25 \mu \mathrm{W}$ (confocal image) and $7 \mu \mathrm{W}$ (STED image) for $A$ excitation (640 $\mathrm{nm}$ ), and a $775 \mathrm{~nm}$ laser pulse energy of $8 \mathrm{~nJ}$. In all cases, the pixel size was set to $20 \mathrm{~nm}$, and

the pixel dwell time values were $0.1 \mathrm{~ms}$ (confocal) and $0.5 \mathrm{~ms}$ (STED). The ratio $\frac{d_{D}^{A}}{d_{D}^{D}}$, which is needed for $F$ calculation and accounts for the cross-talk of $D$ emission into $A$ channel, was measured in samples that were singly labeled with ATTO 594 (being the targets either $\alpha$-tyrtubulin or actin).

\section{Imaging Work-Flow}

The imaging work-flow is depicted in Supplementary Figure 4. It includes both imaging and post-processing steps. The pipeline includes acquiring images in both singly and doubly labeled samples. Details of each action can be found in the figure caption. 


\section{Acquisition sequence}

1) Confocal image, A excitation, $A$ detection, low excitation intensity.

2) STED image, $A$ excitation, $A$ detection $\left(F_{A}^{A}\right)$.

3) Confocal image, $A$ excitation, $A$ detection, low excitation intensity.

4) STED image, $D$ excitation, $A$ and $D$ detection $\left(F_{D}^{D}\right.$ and $\left.F_{A}^{D}\right)$.
Doubly labelled sample

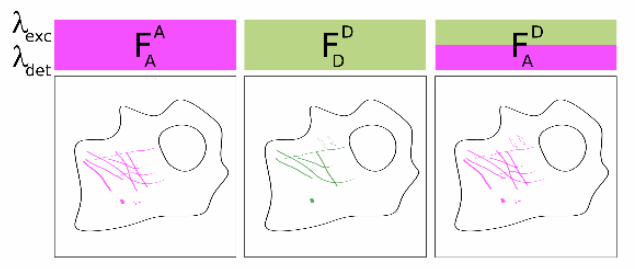

\section{Post-processing sequence}

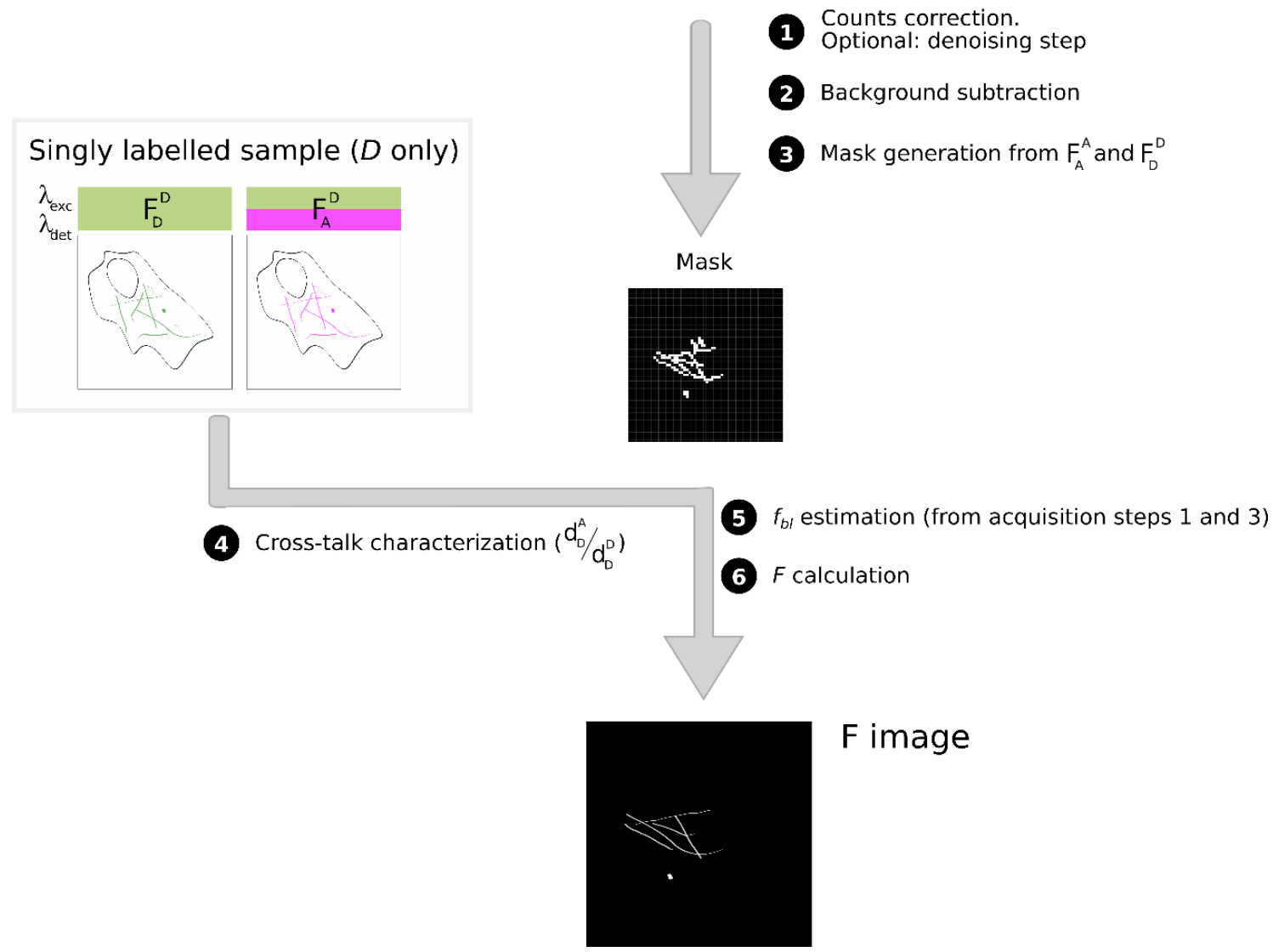

Supplementary Figure 4: STED-FRET Work-Flow. The acquisition sequence for samples labelled with both $D$ and $A$ is shown at the top. It includes obtaining two confocal images under $A$ excitation (Steps 1 and 3), acquired before and after the $F_{A}^{A}$ STED measurement (Step 2). The intensity ratio between these images allows calculating the photobleaching correction factor $f_{b l}$. Finally, Step 4) represents the acquisition of $F_{A}^{D}$ and $F_{D}^{D}$ STED images. The post-processing of the raw data begins with the conversion of counts to photons by considering the wavelength- and countrate-dependent quantum efficiency of the detectors. Subsequently, a denoising step can be performed. The third post-processing action is the background subtraction. Next, a binary mask needs to be obtained, in order to only calculate $F$ in those pixel where we can ensure that there are $A$ and $D$ molecules. For this, an intensity threshold 
has to be set. In this work, we have chosen a relative threshold value for each analysed image, which is

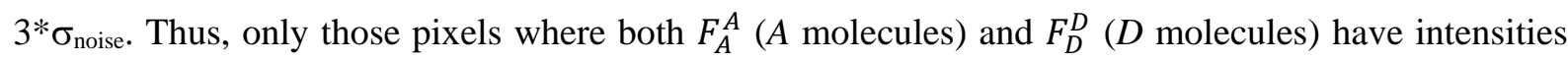
above their respective thresholds will be analysed. Before calculating $F$ pixelwise, the cross-talk of $D$ into $A$ channel has to be calculated. This can be attained using samples singly labelled with $D$, by measuring the ratio of the corrected and background-subtracted emissions obtained in each channel. Finally, $F$ can be calculated in every pixel included within the mask using Eq. 10, achieving this way the $F$ image.

Data acquisition consists of two steps. First, using $A$ excitation, the $F_{A}^{A}$ STED image is obtained. Second, using $D$ excitation the $F_{D}^{D}$ and $F_{A}^{D}$ STED images are acquired. Some photobleaching of $D$ molecules is likely to occur during the first STED acquisition $\left(F_{A}^{A}\right)$. If this happens, a correction factor $\left(f_{b l}\right)$ can accompany $F_{A}^{A}$ in eq. (3), to equalize the concentration of donor molecules in both acquisition steps:

$$
F=\frac{F_{A}^{D}}{F_{D}^{D}}-\frac{I^{D} \sigma_{A}^{D} F_{A}^{A} f_{b l}}{I^{A} \sigma_{A}^{A} F_{D}^{D}}-\frac{d_{D}^{A}}{d_{D}^{D}}
$$

$f_{b l}$ is obtained by acquiring confocal images (STED laser off) before and after the $F_{A}^{A}$ acquisition, using low laser power. $f_{b l}$ is obtained as the average intensity ratio of the confocal images acquired after and before the STED imaging. Before computing $F$, the intensity of the images must be corrected by considering the wavelength- and count-rate dependent quantum efficiency of the detectors. Optionally, denoising algorithms can be applied to the input images. In the biological examples presented below, we applied a deep-learning denoising algorithm ${ }^{6}$ that introduces negligible distortions in the intensity and resolution (Further details in Supplementary Section 7 in the Supporting Information). Next, the background is subtracted and a mask is applied to filter out pixels for which either the donor $\left(F_{D}^{D}\right)$ or the acceptor $\left(F_{A}^{A}\right)$ signal is too low. By doing this, one limits the analysis to regions where the FRET estimation problem is well defined ( $D$ and $A$ molecules are present). This is also a usual procedure for conventional FRET imaging ${ }^{7}$. The selection mask is defined by an intensity threshold set just above the noise level; we applied a threshold of 3 standard deviations of the noise. 


\section{Supplementary Section 7. Denoising}

Reducing the noise of input images for intensity-based FRET imaging may be advantageous for the following reason. $F$ is calculated from three terms (equation 4 of the main text), from which two include the ratio of images. Thus, using noisy input images redounds in even noisier $F$ images. Such $F$ variability due to noise does not reflect the real FRET scenario. Hence, an efficient denoising procedure helps to achieve a more realistic FRET analysis. This is a general issue in intensity-based FRET imaging (see e.g. ref. ${ }^{7}$ ). Optionally, the acquired images can be subjected to a denoising process to improve the SNR. We note that denoising may be advantageous for the intensity-based STED-FRET as long as the denoising algorithm does not distort the intensity levels or the resolution. In Supplementary Figure 5 we show representative raw and denoised images using a deep learning algorithm described in ${ }^{6}$. The intensity profiles are shown to illustrate that this denoising algorithm introduces only negligible alterations to local intensity values and that it does not deteriorate the achieved resolution. The example images shown include all the biological targets shown in the manuscript (microtubules, actin and spectrin), and the performance of denoising both emission channels. 

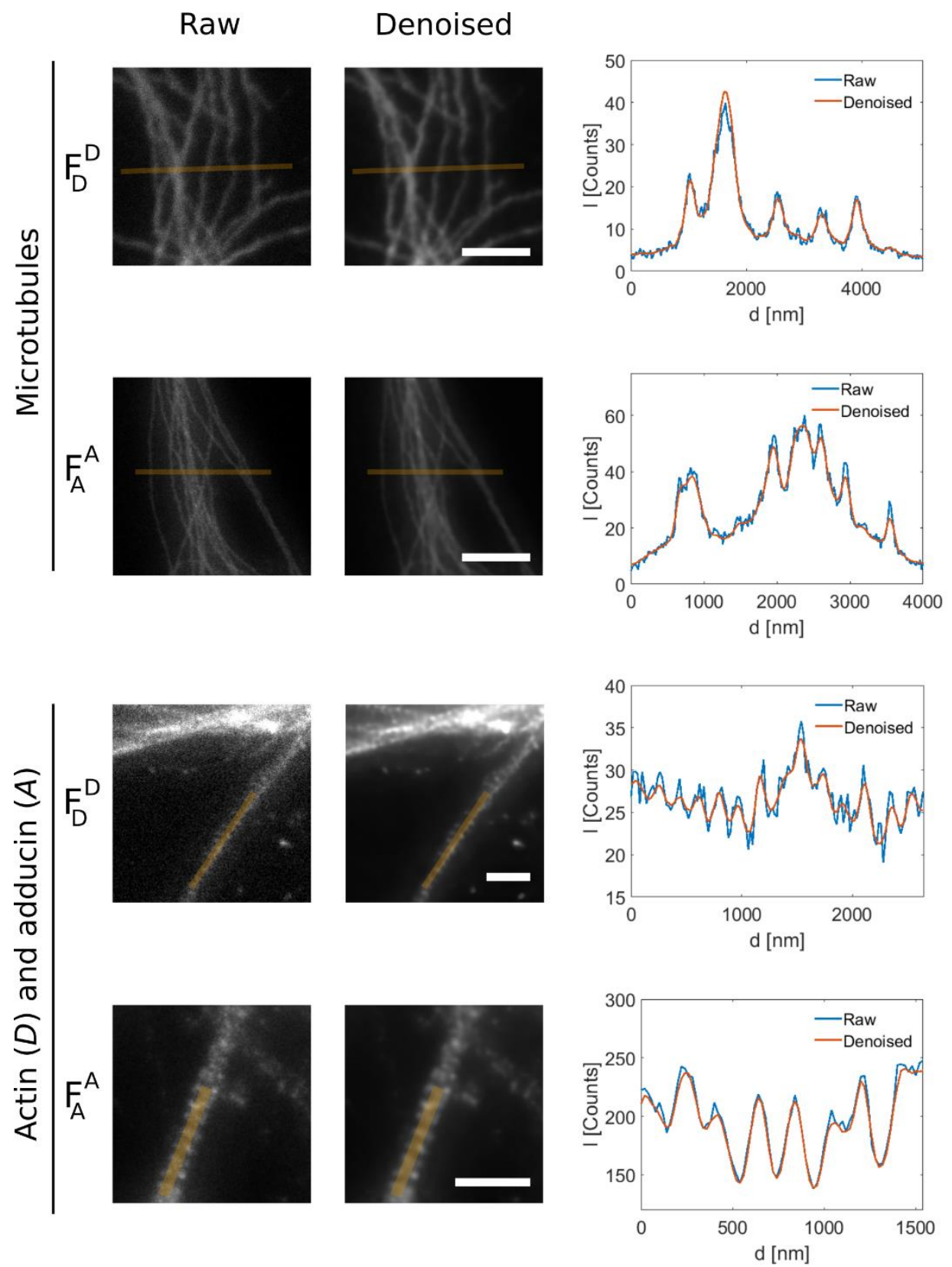

Supplementary Figure 5. Intensity profile comparison between raw and denoised images. Raw and denoised example images of microtubules $\left(F_{D}^{D}\right.$ and $\left.F_{A}^{A}\right)$, actin $\left(F_{D}^{D}\right)$ and adducin $\left(F_{A}^{A}\right)$ are shown on the left. On the right, the intensity profiles from the regions marked in orange on the raw and denoised images are depicted. Scalebars: $2 \mu \mathrm{m}$ (microtubules images), $1 \mu \mathrm{m}$ (actin and adducin images). 


\section{Supplementary Section 8. Evaluation of STED-FRET through simulations}

Simulations shown in Supplementary Figures 6 and 7 were carried out using Gaussian effective PSFs with standard deviations of $105 \mathrm{~nm}$ for the diffraction-limited case and $20 \mathrm{~nm}$ for STEDFRET. Photon counts for $D$ and $A$ molecules were simulated using a Poisson distribution. In the absence of FRET, the average values were 3000 and 717 counts for $D$ and $A$ molecules, respectively ( $A$ counts representing its direct excitation under only $D$ excitation). Background was normally distributed with an average of 20 counts per pixel and $\sigma=2$ counts (STED simulations), and 4 counts per pixel and $\sigma=0.4$ counts (confocal simulations). The values of quantum efficiency, relative absorption cross-sections, and $d_{D}^{A} / d_{D}^{D}$ were: $\phi_{A}=0.65$, $\phi_{D}($ absence of FRET $)=0.85, \phi_{t}=0.75, d_{D}^{A}=d_{D}^{D}$, and $1.25 \sigma_{D}^{D}=3.8 \sigma_{A}^{D}=\sigma_{A}^{A}$. Thus, in the presence of FRET the photon counts for $D$ and $A$ molecules changed to 750 and 2437 counts, respectively.

Supplementary Figures $6 \mathrm{a}, 6 \mathrm{~b}$, and $6 \mathrm{c}$ show three different patterns of $D$ - $A$ molecules forming arrays of positive and negative FRET, along with their corresponding confocal and STED $F$ images. Two kinds of $D-A$ spots were considered, F0 and F1, each containing $9 D$ and $9 A$ molecules, but differently distributed. The F0 spots are configured to deliver negligible FRET; all molecules are $>10 \mathrm{~nm}$ apart from each other (sketch shown in Supplementary Figure 6a). The F1 spots are made to deliver efficient FRET. $D$ and $A$ molecules are placed in pairs with a separation of $3 \mathrm{~nm}$ (sketch shown in Supplementary Figure 6b); an $F$ value of 4 is assigned to the configuration. Simulated diffraction-limited and STED $F_{D}^{D}, F_{A}^{D}$ and $F_{A}^{A}$ images are shown in the Methods section and in Supplementary Figure 7.

The simulations shown in Supplementary Figures $6 a$ and $6 b$ were made with an array of $7 \times 13$ $D$ - $A$ spots. Horizontally, the spots were separated by $500 \mathrm{~nm}$ and were therefore well resolved in both the diffraction-limited and STED images. Vertically, the spots were separated by 150 $\mathrm{nm}$ and could only be clearly resolved through STED imaging. Gaussian effective PSFs with $\sigma$ values of $105 \mathrm{~nm}$ for the diffraction-limited case and $20 \mathrm{~nm}$ for STED-FRET were used (FWHM of $247 \mathrm{~nm}$ and $47 \mathrm{~nm}$, respectively; sketch shown in Supplementary Figure 6a). The 
simulation in Supplementary Figure 6a was made only with F0 spots. As expected, both confocal and STED $F$ images present values around zero. Ideally, in the absence of FRET, $F$ should take on a value of zero. However, variations in the photon-counts product of the Poisson emission and background noise lead to variability of $F$. The negative control images present a distribution of $F$ values that is nearly normal, centered around zero with a FWHM of $\sim 1.1$ (Supplementary Figure 6d). In real samples, $F$ may present a larger variability due to local differences in $D$ and $A$ concentrations. An additional contribution may be present in STEDFRET. Due to the small size of the effective PSF, the number of $D$ and $A$ molecules addressed are typically low. Thus, variations in the individual emission and/or absorption spectra of the molecules may also contribute to the variability of $F$. For these reasons, a negative control is required in order to characterize these variations and determine the minimum $F$ value that reports actual $D-A$ molecular interaction.

The simulation shown in Supplementary Figure $6 \mathrm{~b}$ includes F1 spots forming a triangle surrounded by F0 spots. This simulation is designed to evaluate the PSF averaging of $F$ at the borders. STED-FRET clearly resolves all the FRET positive spots and determines a value of $F$ close to the true value of 4 for all. In the diffraction-limited FRET image, the FRET positive spots are not resolved vertically, as expected. Importantly, the vertical stripes also merge horizontally in the $F$ image. The reason for this result is that even though the intensity of the input images drops between stripes (Supplementary Figure 6), their intensity relations are maintained, and above the threshold to be in included the mask to compute $F$. Vertically, a gradual reduction of $F$ at the borders is observed due to the PSF averaging of regions of no FRET (F0 spots), except for the last column which is composed only of F1 spots. This is further illustrated in the distributions of $F$ shown in Supplementary Figure 6d, for the areas marked as 1 to 3 in the images of Supplementary Figure 6b. For region 3, the $F$ histograms for STEDFRET and confocal-FRET are practically indistinguishable because region 3 only includes F1 spots and there is no averaging. Regions 1 and 2 include F1 spots in the center and F0 spots above and below. Because the separation between F1 and F0 spots is smaller than confocal PSF, confocal-FRET becomes less accurate due to averaging at the vertical borders. The diffraction-limited imaging of regions 1 and 2 yields a wide distribution of $F$ values between 0 and 5. In contrast, STED-FRET imaging is able to resolve the individual F0 and F1 spots, and 
delivers a bimodal distribution of $F$ values with peaks corresponding well to the expected values $(F=4, F=0)$.

Supplementary Figure $6 \mathrm{c}$ shows a third simulation, more relevant to interpret real sample situations, using an array of sub-diffraction dimensions where F0 and F1 spots were randomly interspersed. The horizontal inter-spot distance was $70 \mathrm{~nm}$, while in the vertical direction it was $190 \mathrm{~nm}$, i.e. the spots were only resolvable through STED in both directions. Remarkably, STED-FRET is also capable of resolving both F0 and F1 spots, and accurately measuring their ground-truth values (see the bimodal distribution in Supplementary Figure 6d, region 4). In contrast, for diffraction-limited FRET the $F=4$ value is never reached, due to the averaging effect produced when many F0 and F1 spots are simultaneously detected within the same diffraction-limited PSF. Even though some regions appear to have higher $F$ values than others, the boundaries between low and high $F$ regions are blurred in comparison to the STED-FRET approach, resulting in a poorer image-based FRET analysis. 
a) Negative control array

d) $F$ distributions

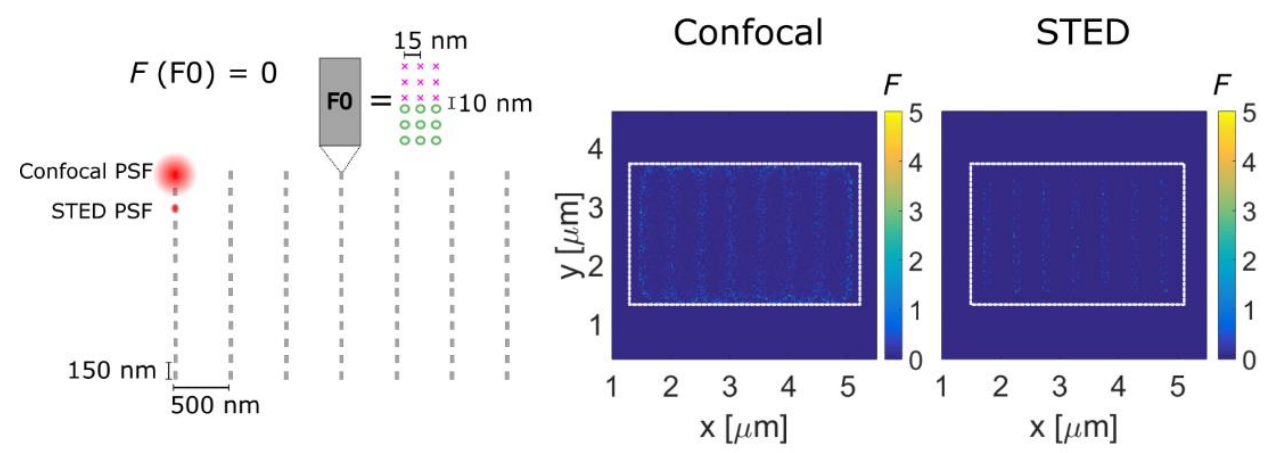

b) Positive FRET array - Averaging at borders
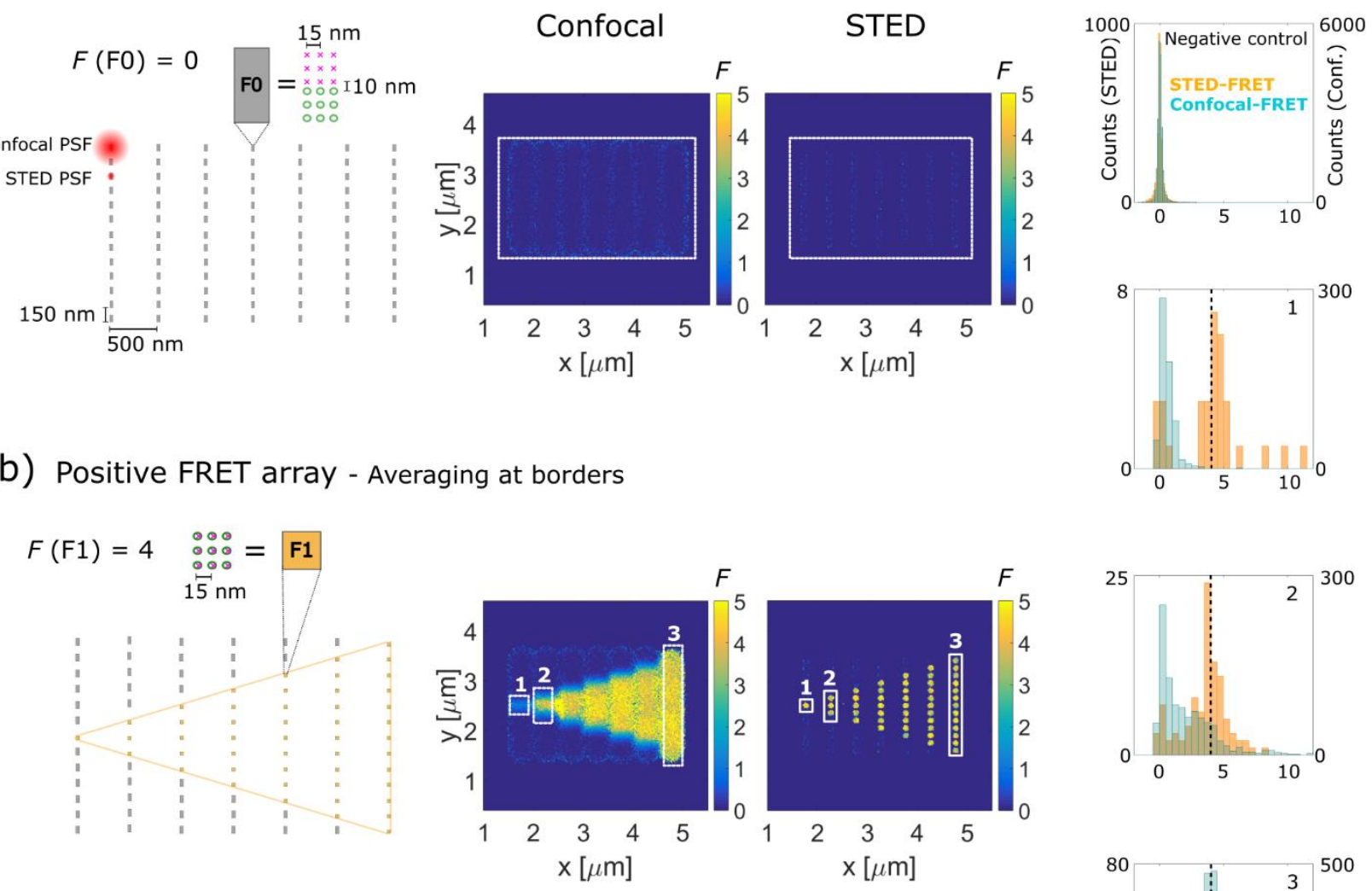

c) Positive FRET array - Random averaging
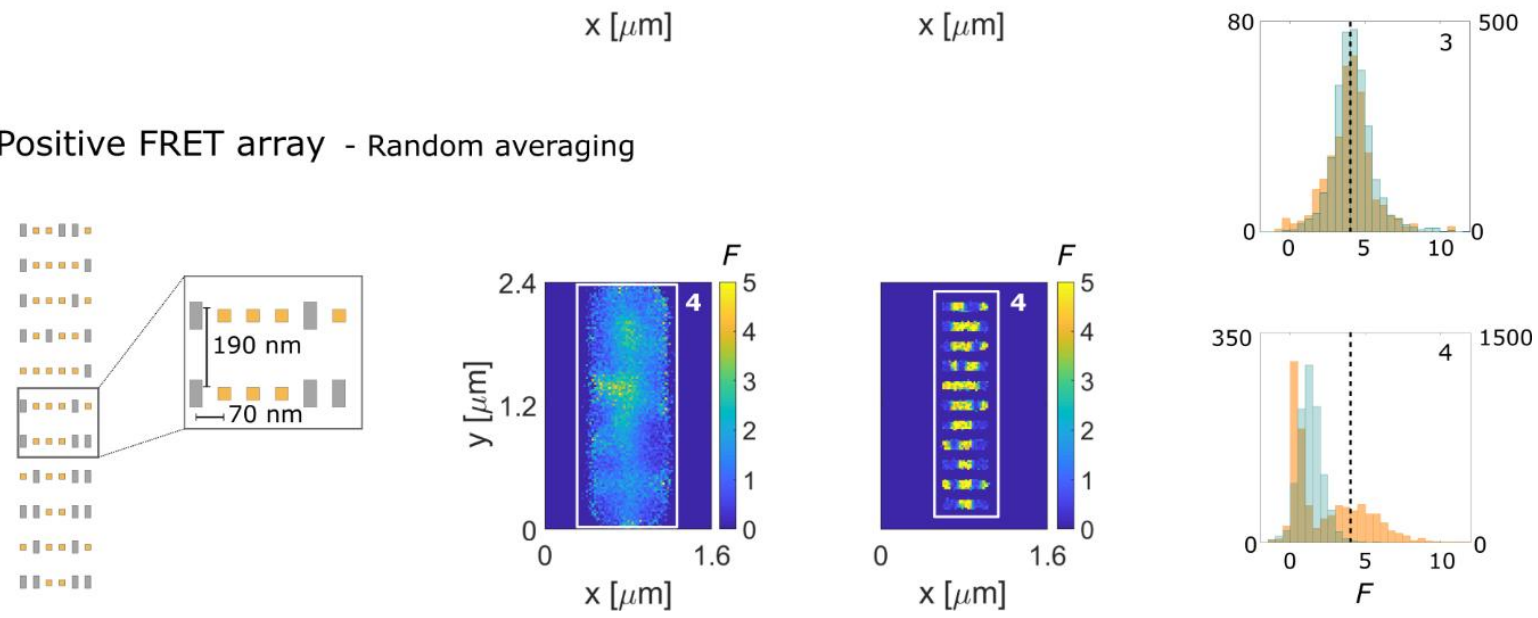

Supplementary Figure 6. Comparison between diffraction-limited and STED-FRET analysis performances on simulated data. a) Left: Schematic distribution of F0 blocks ( 9 donor and 9 acceptor molecules; $F=0$ ) in a $7 \times 13$ stripes array. The centers of F0 blocks are separated $150 \mathrm{~nm}$ vertically and $500 \mathrm{~nm}$ horizontally. Right: $F$ images computed by diffraction limited-FRET (left) and STED-FRET (right). b) Left: Schematic distribution of F0 and F1 (9 donor and 9 acceptor molecules; $F=4$ ) blocks in the same 7x13 stripes array as the one shown in a), but with F1 blocks occupying the triangular region delimited by the yellow lines. Right: Diffraction limited-FRET (left) and STED-FRET (right) images obtained from $F$. c) Left: Distribution of F0 and F1 blocks in a stripes array with separations of $70 \mathrm{~nm}$ 
horizontally and $190 \mathrm{~nm}$ vertically. F0 and F1 randomly occupy the positions of the array. Right: Diffraction-limited and STED-FRET images (left and right, respectively). d) Histograms of $F$ for the different simulated conditions. Orange: STED-FRET; cyan: diffraction limited-FRET. From top to bottom: negative control (values obtained from the rectangular region marked in a); regions 1-3 (correspond to the marked regions in b); and region 4 (corresponds to the marked regions in c). Dashed lines indicate the ground-truth value for F1 $(F=4)$.

a) Negative control array

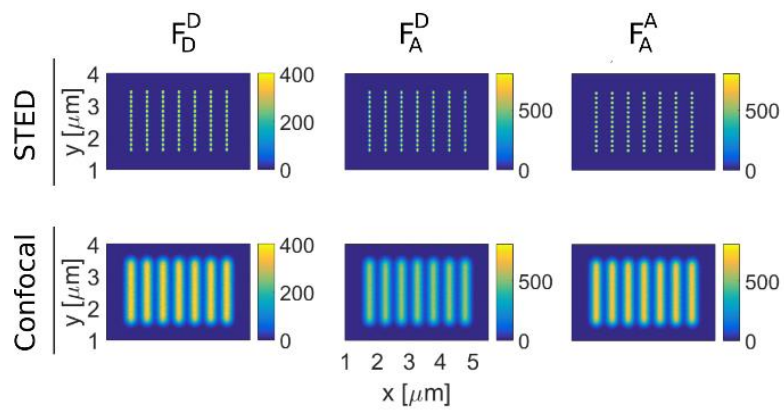

C) Positive FRET array (random averaging)

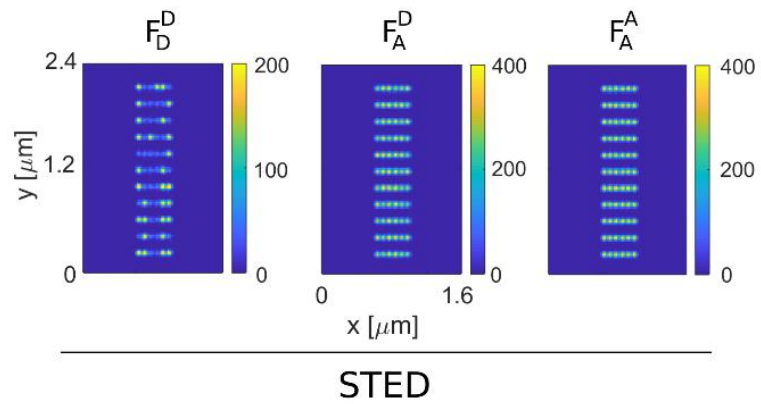

b) Positive FRET array (averaging at borders)

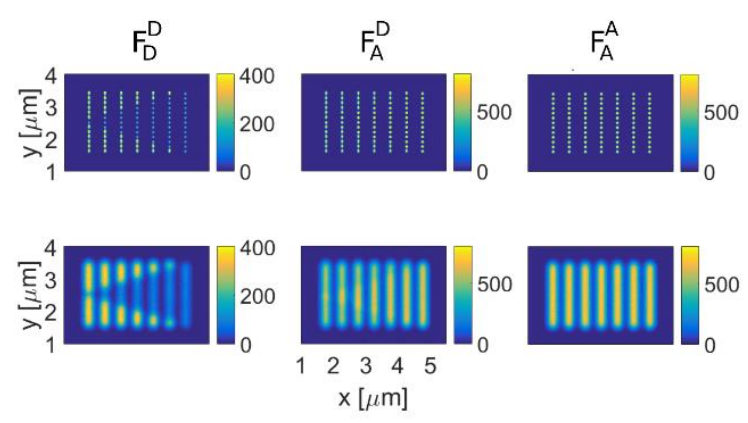

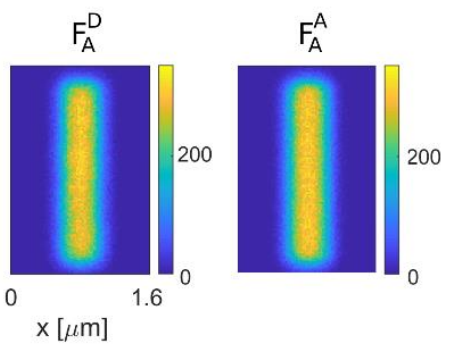

Confocal

Supplementary Figure 7: Masked intensity images of the simulated data. $F_{D}^{D}, F_{A}^{D}$, and $F_{A}^{A}$ images obtained for the different simulations after applying a binary mask based on an intensity threshold, and subtracting the background mean value. For a) and b), the STED images are depicted at the top and the confocal images at the bottom; for c), STED images are shown on the left and confocal images on the right. a) Images of the negative control simulation shown in Supplementary Figure 6a (array of F0 blocks). b) Images of the positive FRET array depicted in Supplementary Figure 6b, where F1 blocks are organized within a triangular region, surrounded by F0 blocks. c) Images of the positive FRET shown in Supplementary Figure 6c, where F0 and F1 blocks are randomly interspersed. 


\section{Supplementary Section 9. F distributions from Figure 4.}

Example neuron 1
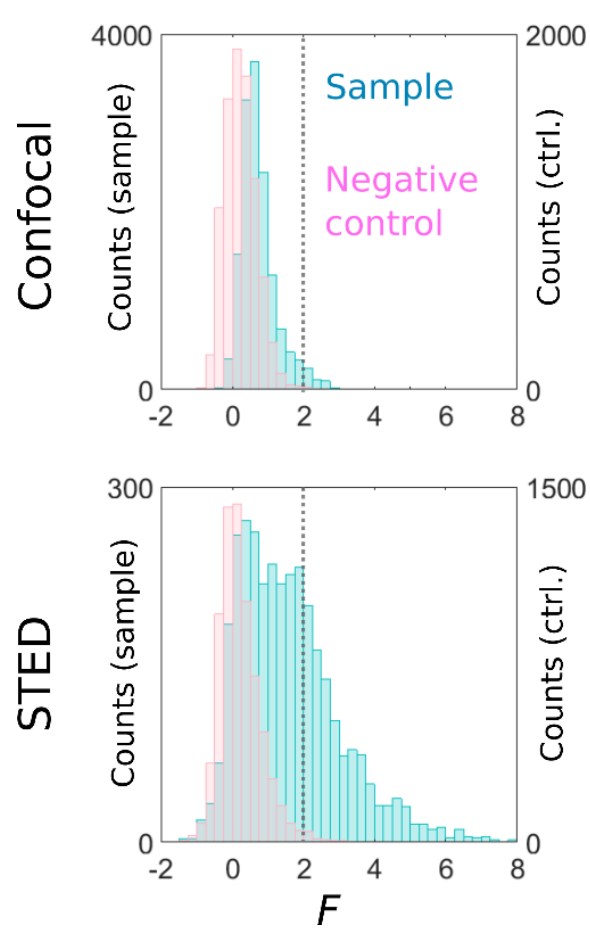

Example neuron 2
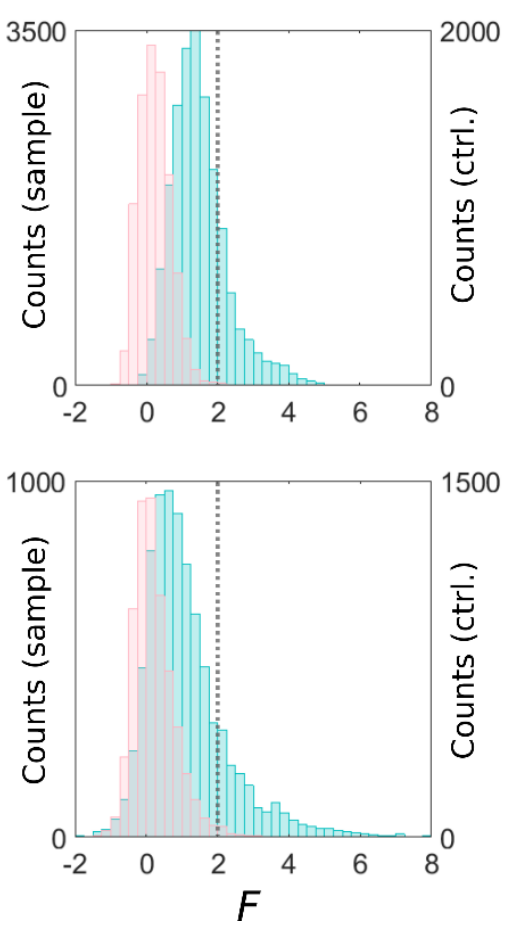

Supplementary Figure 8. $\boldsymbol{F}$ histograms of example neurons from Figure 4. Confocal (top) and STED (bottom) $F$ histograms obtained for example neuron 1 (left) and example neuron 2 (right) from Figure 4. The sample histograms are colored in cyan, while the negative control histograms are plotted in pink. The negative control distributions were obtained from microtubule images as explained in the main text. The $F$ threshold value $(F=2)$ is shown in each plot with a dotted grey line.

\section{Supplementary Section 10. Preparation of biological samples}

\section{Primary neuron culture and cell lines}

Primary hippocampal neuronal cultures were prepared from mouse embryos (CD1 strain) at embryonic day 17, following the general guidelines of the National Institute of Health (NIH, 
USA) and approval of the National Department of Animal Care and Health (SENASA, Argentina), and cultured in Neurobasal medium (Gibco) supplemented with 5 mM GlutaMAXI (Gibco) and 2\% B27 supplement (Gibco) at $37{ }^{\circ} \mathrm{C}$ and $5 \% \mathrm{CO}_{2}$. Neurons were seeded at a density of 125 cells $/ \mathrm{mm}^{2}$ on cell culture 24 wells plates with round $12 \mathrm{~mm}$ diameter glass coverslips, \#1.5 thickness (ThermoFischer), and incubated for 3 days (microtubules staining) or 28 days (actin and adducin staining). To increase cell attachment, coverslips were previously coated with $0.05 \mathrm{mg} / \mathrm{mL}$ poly-L-lysine (overnight at $37^{\circ} \mathrm{C}$ ) (Sigma Aldrich) and $1 \mu \mathrm{g} / \mu \mathrm{L}$ Laminin $\left(3 \mathrm{~h}\right.$ at $\left.37^{\circ} \mathrm{C}\right)$ (Sigma Aldrich).

\section{Fixation and permeabilization of neuron samples}

Cultured neurons were fixed and permeabilized in PHEM buffer (60 mM PIPES, $25 \mathrm{mM}$ HEPES, 5 mM EGTA, 1 mM MgCl 2 , pH 7.0), supplemented with $0.25 \%$ glutaraldehyde, $3.7 \%$ paraformaldehyde, $3.7 \%$ sucrose and $0.1 \%$ Triton X-100, for 20 min at room temperature. Autofluorescence was quenched by incubating the samples in $0.1 \mathrm{M}$ glycine in PBS for 15 minutes followed by $3 \times$ washes with PBS. The fixed and quenched samples were blocked with 5\% BSA in PBS containing $0.01 \%$ Triton $\mathrm{X}-100$ for $1 \mathrm{~h}$.

\section{Microtubule immunostaining}

For microtubule staining, $\alpha$-tyr-tubulin was labeled with a mouse monoclonal primary antibody anti- $\alpha$-tyrosinated Tubulin (Clone TUB-1A2, Sigma Aldrich) for $1 \mathrm{~h}$ at room temperature using a 1:200 dilution in 5\% BSA in PBS, followed by $3 \times$ washes with PBS. Alternative secondary antibody staining was performed by incubating at a 1:250 dilution in 5\% BSA in PBS for $1 \mathrm{~h}$ at room temperature either with: a) a goat anti-mouse IgG ATTO 594 antibody (76085, Sigma Aldrich); b) a goat anti-mouse IgG ATTO 647N antibody (50185, Sigma Aldrich); or c) Both of them. This incubation was followed by $3 \times$ washes with PBS.

\section{Actin and adducin immunostaining}

To identify actin rings on neurons we used a co-staining strategy labeling actin and adducin together. Adducin was labeled with a rabbit polyclonal primary antibody anti-alpha-adducin 
(Abcam ab51130) for $1 \mathrm{~h}$ at room temperature using a 1:200 dilution in 5\% BSA in PBS. Secondary antibody staining was performed by incubating with a goat anti-rabbit IgG ATTO $647 \mathrm{~N}$ antibody (50185, Sigma Aldrich) at a 1:250 dilution in 5\% BSA in PBS for $1 \mathrm{~h}$ at room temperature. After secondary antibody incubation, actin was labeled with Phalloidin-ATTO 594 (51927, Sigma Aldrich) at a $10 \mu \mathrm{L} / \mathrm{mL}$ in PBS for $1 \mathrm{~h}$ at room temperature followed by $3 \times$ washes with PBS. Additionally, for cross-talk measurements, samples were prepared where only actin was labeled, using the corresponding steps of the co-immunostaining.

\section{Supplementary Section 11. DNA sequences used for building the DNA origami}

\begin{tabular}{|l|l|l|l|}
\hline Start & End & Sequence & \\
\hline $0[76]$ & $0[54]$ & GACTTTCTCCGTGGCGCGGTTG & \\
\hline $11[93]$ & $0[77]$ & GCTCAAGTTGGGTAACGGGCGGAAAAATTTGTGAGAGATA & \\
\hline $0[118]$ & $0[96]$ & CGAGTAACAACCGTTTACCAGTC & \\
\hline $11[135]$ & $0[119]$ & TAAAGGATTGTATAAGCGCACAAACGACATTAAATGTGAG & \\
\hline $0[160]$ & $0[138]$ & GATAAAAATTTTTAGCCAGCTTT & \\
\hline $11[177]$ & $0[161]$ & TGCGTACTAATAGTAGTTGAAATGCATATTTCAACGCAAG & \\
\hline $0[202]$ & $0[180]$ & GACCGGAAGCAATTGCGGGAGAA & \\
\hline $11[219]$ & $0[203]$ & GTGCCTGCTTTAAACAGGGAGAGAGTTTCAAAGCGAACCA & \\
\hline $0[244]$ & $0[222]$ & AAAAATCTACGTGCGTTTTAATT & \\
\hline $11[261]$ & $0[245]$ & CGGTTAACAAAGCTGCTGTAACAACAAGGACGTTGGGAAG & \\
\hline $0[286]$ & $0[264]$ & AAAACGAAAGAGGCTCATTATAC & \\
\hline $11[303]$ & $0[287]$ & GTGAGTTAAAGGCCGCTGACACTCATGAAGGCACCAACCT & \\
\hline $0[328]$ & $0[306]$ & TTGTCGTCTTTCTACGTAATGCC & \\
\hline $11[345]$ & $0[329]$ & GAGAGCCTCAGAACCGCATTTTCTGTAACGATCTAAAGTT & \\
\hline $0[370]$ & $0[348]$ & GCGTCATACATGCCCTCATAGTT & \\
\hline $11[387]$ & $0[371]$ & GGCGACACCACCCTCAGGTTGTACTGTACCGTTCCAGTAA & \\
\hline $0[412]$ & $0[390]$ & TCACCGTCACCGGCGCAGTCTCT & \\
\hline $11[429]$ & $0[413]$ & CCCTTCATATAAAAGAACGTAGAGCCTTAAAGGTGAATTA & \\
\hline $0[454]$ & $0[432]$ & AGACGGGAGAATTGACGGAAATT & \\
\hline $11[471]$ & $0[455]$ & TGTTCCAACGCTAACGAACAAGTCAGCAGGGAAGCGCATT & \\
\hline $0[496]$ & $0[474]$ & TCCCATCCTAATGAGAATAACAT & \\
\hline $11[513]$ & $0[497]$ & CTCCAATTTAGGCAGAGACAATCAATCAAGAAAAATAATA & \\
\hline
\end{tabular}




\begin{tabular}{|c|c|c|}
\hline $0[538]$ & $0[516]$ & TTAGGTTGGGTTATAGATAAGTC \\
\hline $11[555]$ & $0[539]$ & CCCACATGTGAGTGAATAACTGATGCTTTTAACCTCCGGC \\
\hline $0[580]$ & $0[558]$ & TAGAACCTACCAGTCTGAGAGAC \\
\hline 11[597] & $0[581]$ & $\begin{array}{l}\text { GAGGTAACGTTATTAATTTTAAAACAAATAATGGAAGGGT } \\
\end{array}$ \\
\hline $0[622]$ & $0[600]$ & AAGATAAAACAGTTGGATTATAC \\
\hline 11[639] & 0 [623] & CCGATAATAAAAGGGACTTAACACCGCGAACCACCAGCAG \\
\hline $0[664]$ & $0[642]$ & GATTTTAGACAGGCATTAAAAATA \\
\hline $3[667]$ & $0[665]$ & GGCGCCCCGCCGAATCCTGAGAAGTGAGGCCGATTAAAGG \\
\hline 6[69] & $2[56]$ & AAAAGTGTCAGCAACAATTGCAGGCGCT \\
\hline $6[111]$ & $2[98]$ & TCAGGTGAAATTTCTACGGAAACAATCG \\
\hline $6[153]$ & $2[140]$ & TAAATCGGTTGGTGCACATCAAAAATAA \\
\hline $6[195]$ & $2[182]$ & TGCAACTCAAAAGGCCGTACCAAAAACA \\
\hline $6[237]$ & $2[224]$ & AAGAGATTCATTTTGTTTAAGAGGAAGC \\
\hline $6[279]$ & $2[266]$ & CATGTCAGAGATTTGATGTGAATTACCT \\
\hline $6[321]$ & 2 2[308] & AAATCCCCGAAACAATTCATGAGGAAGT \\
\hline $6[363]$ & $2[350]$ & TGAAATTGTTTCAGGGAACTACAACGCC \\
\hline 6[405] & 2 2[392] & CAAGTGCTGAGTAAGAAAATAAATCCTC \\
\hline 6[447] & 2 2[434] & TTACCTCTTAGCAAATTTCAACCGATTG \\
\hline 6[489] & 2 [476] & AATCATAATAACCCGGCGTCAAAAATGA \\
\hline $6[531]$ & 2 2[518] & GACCGTCGAACGGGGAAGCTAATGCAGA \\
\hline $6[573]$ & $2[560]$ & TGATTTAGAAAACTCAAGAGTCAATAGT \\
\hline $6[615]$ & 2 2[602] & GTCAGTCGTTTAACGAGATGGCAATTCA \\
\hline $6[657]$ & 2 2[644] & TGCCTGAACAGCAAATGAATGCGCGAACT \\
\hline $2[55]$ & $3[55]$ & TTCGCCATAAACTCTGGAGGTGTCCAGC \\
\hline $3[56]$ & $3[78]$ & ATCAGCGGGGTCAGCTTTCAGAG \\
\hline 2 2[97] & $3[97]$ & GCGAAAGACGCAAAGCCGCCACGGGAAC \\
\hline $3[98]$ & $3[120]$ & GGATAACCTCACAATTTTTGTTA \\
\hline 2 [139] & $3[139]$ & TTCGCGGATTGATTGCTCATTTTTTAAC \\
\hline $3[140]$ & $3[162]$ & CAATAGGAACGCAAATTAAGCAA \\
\hline $2[181]$ & $3[181]$ & TTATGGCCTGAGCACCTCAGAGCATAAA \\
\hline $3[182]$ & $3[204]$ & GCTAAATCGGTTTGACTATTATA \\
\hline 2 2223] & $3[223]$ & CCGAACTTTAATAAAAGCAAAGCGGATT \\
\hline $3[224]$ & $3[246]$ & GCATCAAAAAGAAGTAAATTGGG \\
\hline 2 2[265] & $3[265]$ & TATGCATTACAGAGGATGGTTTAATTTC \\
\hline $3[266]$ & $3[288]$ & AACTTTAATCATGGGTAGCAACG \\
\hline 2 2[307] & $3[307]$ & TTCCATTGACCCAAAGAGGCTTTGAGGA \\
\hline $3[308]$ & $3[330]$ & CTAAAGACTTTTAGGAACCCATG \\
\hline 2 [349] & $3[349]$ & TGTAGGGGATTTAGTAACACTGAGTTTC \\
\hline $3[350]$ & $3[372]$ & GTCACCAGTACAAGGTTGAGGCA \\
\hline $2[391]$ & $3[391]$ & ATTAAAATAAGTGCGACGATTGGCCTTG \\
\hline
\end{tabular}




\begin{tabular}{|c|c|c|}
\hline 3[392] & $3[414]$ & ATATTCACAAACAAATTCATATG \\
\hline 2[433] & $3[433]$ & AGGGACAAAATCTTCCAGCGCCAAAGAC \\
\hline $3[434]$ & $3[456]$ & AAAAGGGCGACAATTATTTATCC \\
\hline 2[475] & $3[475]$ & AAATAGGTAATTTACAAATAAGAAACGA \\
\hline $3[476]$ & $3[498]$ & TTTTTTGTTTAATAAAGTAATTC \\
\hline 2 2[517] & $3[517]$ & ACGCGTCGGCTGTAAGACGACGACAATA \\
\hline $3[518]$ & $3[540]$ & AACAACATGTTCATCCTTGAAAA \\
\hline 2 2[559] & $3[559]$ & GAATTATCCAATAACGATAGCTTAGATT \\
\hline $3[560]$ & $3[582]$ & AAGACGCTGAGACCAGAAGGAGC \\
\hline $2[601]$ & $3[601]$ & TCAATAATAAAGTGTATCATCATATTCC \\
\hline $3[602]$ & $3[624]$ & TGATTATCAGATATACGTGGCAC \\
\hline 2 [643] & $3[643]$ & GATAGTGCAACATGATATTTTTGAATGG \\
\hline $3[644]$ & $3[666]$ & CTATTAGTCTTTCGCCGCTACAG \\
\hline 5[77] & $4[84]$ & $\begin{array}{l}\text { AACGTTGTAGAAACAGCGGATAGTTGGGCGGTTGT } \\
\end{array}$ \\
\hline $4[125]$ & $4[103]$ & GTTTGAGGGGACCTCATTTGCCG \\
\hline $5[119]$ & $4[126]$ & CATAATATTCCGTAATGGGATCCGTGCATCTGCCA \\
\hline $4[167]$ & $4[145]$ & CAATATGATATTGATGGGCGCAT \\
\hline $5[161]$ & $4[168]$ & GTATACAGGTAATGTGTAGGTAGTCAAATCACCAT \\
\hline $4[209]$ & $4[187]$ & AATGCTGTAGCTGAGAAAGGCCG \\
\hline 5 [203] & $4[210]$ & TGTAAATCATGCTCCTTTTGATAATTGCTGAATAT \\
\hline 5 [245] & $4[252]$ & CGCCTGACGGTAGAAAGATTCTAATGCAGATACAT \\
\hline 4 [293] & $4[271]$ & AAATTGTGTCGAGAATACCACAT \\
\hline 5 [287] & $4[294]$ & GCGCAGCGACCAGCGATTATATATCATCGCCTGAT \\
\hline $4[335]$ & $4[313]$ & ATTGCGAATAATGTACAACGGAG \\
\hline 5 [329] & $4[336]$ & TTCATTTTCTGCTAAACAACTGAACAACTAAAGGA \\
\hline $4[377]$ & $4[355]$ & CTATTTCGGAACGAGTGAGAATA \\
\hline $5[371]$ & $4[378]$ & ATCAGAGCCTTTAACGGGGTCTTAATGCCCCCTGC \\
\hline $4[419]$ & 4 4[397] & GCAGCACCGTAAGTGCCCGTATA \\
\hline 5 [413] & $4[420]$ & AGAGTTTATACCAGTAGCACCTGAAACCATCGATA \\
\hline 5 [455] & $4[462]$ & CATGCCAGTGAGCGCTAATATCCAATAATAAGAGC \\
\hline $4[503]$ & $4[481]$ & AGCAAGCCGTTTAAGAATTGAGT \\
\hline 5[497] & $4[504]$ & TTGAGAATATCTTTCCTTATCACTCATCGAGAACA \\
\hline $4[545]$ & $4[523]$ & TGACCTAAATTTTTAAACCAAGT \\
\hline 5[539] & $4[546]$ & TTCGCTATTCGCAAGACAAAGTTAATTTCATCTTC \\
\hline $4[587]$ & $4[565]$ & CATCGGGAGAAATTCAAATATAT \\
\hline $5[581]$ & $4[588]$ & ACATCATTTAAATTGCGTAGAAACAGTACCTTTTA \\
\hline 4[629] & 4 4[607] & CAAATATCAAACCAGATGAATAT \\
\hline 5[623] & $4[630]$ & ATACCCTTCGTGCCACGCTGAACCTTGCTGAACCT \\
\hline $4[671]$ & $4[649]$ & TACTTCTTTGATAAAAATCTAAA \\
\hline 9[63] & $5[76]$ & $\begin{array}{l}\text { TTCACCTAGCGTGGCGGGTGAAGGGATACCAGTGCATAAAAA } \\
\end{array}$ \\
\hline
\end{tabular}




\begin{tabular}{|c|c|c|}
\hline $9[105]$ & $5[118]$ & GTCCGTCCTGCAAGATCGTCGGATTCTCTTCGCATTGGACGA \\
\hline $9[147]$ & $5[160]$ & CATTCAACCCAAAATGTAGAACCCTCATGAATTAGTACAACC \\
\hline $9[189]$ & 5 [202] & GAGCAAGGTGGCATTTACTCCAACAGGTTCTTTACGTCAACA \\
\hline $9[231]$ & $5[244]$ & TTAGTGTGAATCCCTCTAATAAAACGAAAGAACGATGAATTA \\
\hline 9[273] & $5[286]$ & GAAGTCAACCCAAATGGCAAAAGAATACTCGGAACAGAATCC \\
\hline 9[315] & $5[328]$ & CAGATATAGGCTTGAACAGACGTTAGTAAAGCCCAAAAATTT \\
\hline $9[357]$ & $5[370]$ & $\begin{array}{l}\text { TCTTATACTCAGAAAGGCTTTTGATGATATTGACACGCTATT } \\
\end{array}$ \\
\hline 9[399] & $5[412]$ & ATAAGAAGCCACCCAAACTTGAGCCATTATCAATACATCAGT \\
\hline 9[441] & $5[454]$ & TGCCATACATAAAGATTAACTGAACACCAACAGCCGGAATAG \\
\hline 9[483] & $5[496]$ & ATAATGAATCCTGAGATTACGAGCATGTGACAAAAACTTATT \\
\hline $9[525]$ & $5[538]$ & TTTAGCAAACGCCACAATATAACTATATTCCCTTATAAATGG \\
\hline $9[567]$ & $5[580]$ & ATCATTTACATAAAAGTATCAAAATTATAAGAAACTTCAATA \\
\hline $9[609]$ & $5[622]$ & GATGAATAAATCCTGTAGGTGAGGCGGTAGCGTAAGTCCTCA \\
\hline $9[651]$ & $5[664]$ & AATAGCTGTCACACGCAACGGTACGCCAGCGCTTAATGTAGTA \\
\hline 11 [63] & 6[70] & ATAGCTGTTTCCTGGAACGTCCATAACGCCGTAAA \\
\hline $11[105]$ & $6[112]$ & ACACAACATACGAGGGATGTGGCTATTAATCGGCC \\
\hline $11[147]$ & $6[154]$ & TGCCTAATGAGTGAGAAAAGCTCATATGTAGCTGA \\
\hline 11[189] & $6[196]$ & ACTGCCCGCTTTCCTGAAAAGCTATATTTTAAATA \\
\hline $11[231]$ & 6[238] & TTAATGAATCGGCCATTCATTCCAATACGCATAGT \\
\hline 11 [273] & $6[280]$ & TGGGCGCCAGGGTGATTCATTAGAGTAACCTGCTC \\
\hline $11[315]$ & $6[322]$ & ACAGCTGATTGCCCGTCGCTGCGCCCACACGTTGA \\
\hline $11[364]$ & $6[364]$ & GTCCACGCGCCACCTCACCGTTGAAACA \\
\hline 11 [399] & 6[406] & GTTTGATGGTGGTTCAGAACCCCGCCTCACAGAAT \\
\hline 11[441] & 6[448] & AAAAGAATAGCCCGATACATACGCAGTAAGCTATC \\
\hline $11[483]$ & 6[490] & GAACAAGAGTCCACCAATTTTTTAGTTGTCGTAGG \\
\hline $11[525]$ & $6[532]$ & AGGGCGAAAAACCGATTTAACGTAGGGCAAATACC \\
\hline $11[567]$ & 6[574] & ACCATCACCCAAATAAACAGTTCATTTGATTCGCC \\
\hline 11[609] & 6[616] & AGCACTAAATCGGATCGTATTTAGACTTATATCTG \\
\hline $11[651]$ & $6[658]$ & TTGACGGGGAAAGCTTCACCAGAAATGGCATCACT \\
\hline $4[60]$ & $7[62]$ & TCAGAGGTGTGTCGGCCAGAATGAGTGCACTCTGTGGT \\
\hline $7[63]$ & $7[85]$ & $\begin{array}{l}\text { GCCCGCACAGGCGGCCTTTAGTG } \\
\end{array}$ \\
\hline $4[102]$ & $7[104]$ & CCAGCCAAACTTCTGATTGCCGTTTTGGGTAAAGTTAAAC \\
\hline $7[105]$ & $7[127]$ & GAAAGATCGCACTCCAGCCAGCT \\
\hline $4[144]$ & $7[146]$ & CGTAAAGGTCACGAAACCAGGCAATAGCACCGCTTCTGGT \\
\hline $7[147]$ & $7[169]$ & GCTAATGCCGGAGAGGGTAGCTA \\
\hline $4[186]$ & $7[188]$ & GAGACAAAGATTATCAGGTCATTGACGAGAGATCTACAAA \\
\hline 7[189] & $7[211]$ & GGCTAAAGTACGGTGTCTGGAAG \\
\hline $4[228]$ & $7[230]$ & GAGCTTAAGAGGTCCCAATTCTGCAATTCCATATAACAGT \\
\hline $7[231]$ & $7[253]$ & TGCAACACTATCATAACCCTCGT \\
\hline 4 4[270] & $7[272]$ & TCAACATCAGTTAAATAGCGAGAGTGAGACGACGATAAAA \\
\hline
\end{tabular}




\begin{tabular}{|c|c|c|}
\hline 7[273] & 7 [295] & ACTACTTAGCCGGAACGAGGCGC \\
\hline $4[312]$ & $7[314]$ & ATTTGCCAAGCGGAACTGACCAACGAGTCAATCATAAGGG \\
\hline $7[315]$ & $7[337]$ & AATCCAAAAAAAAGGCTCCAAAA \\
\hline $4[354]$ & $7[356]$ & GAAAGTTCAACAATCAGCTTGCTTAGCTTTAATTGTATCG \\
\hline $7[357]$ & $7[379]$ & GTGTATTAAGAGGCTGAGACTCC \\
\hline 4[396] & $7[398]$ & $\begin{array}{l}\text { AACAGAGTGCCTGGGGTTTTGCTCACAGAAGGATTAGGAT } \\
\end{array}$ \\
\hline 7[399] & $7[421]$ & TATTGCCTTTAGCGTCAGACTGT \\
\hline $4[438]$ & $7[440]$ & $\begin{array}{l}\text { ACCAAATTACCAGGTCATAGCCCCGAGTTTTCATCGGCAT } \\
\end{array}$ \\
\hline $7[441]$ & $7[463]$ & TTGAAGCCCTTTTTAAGAAAAGT \\
\hline $4[480]$ & $7[482]$ & TAAGCCAGAGAGCCAGAAGGAAACTCGATAGCCGAACAAA \\
\hline $7[483]$ & $7[505]$ & GTTTACCGCGCCCAATAGCAAGC \\
\hline $4[522]$ & $7[524]$ & $\begin{array}{l}\text { ACCGCATTCCAACGGTATTCTAAGCGAGATATAGAAGGCT } \\
\end{array}$ \\
\hline $7[525]$ & $7[547]$ & TATGTGATAAATAAGGCGTTAAA \\
\hline $4[564]$ & $7[566]$ & TTTAGAACGCGAATTACTAGAAAACTATAAACACCGGAAT \\
\hline $7[567]$ & $7[589]$ & CAGCTTTGAATACCAAGTTACAA \\
\hline $4[606]$ & $7[608]$ & ACAGTTTTTCAGATTTCAATTACCGTCGCAGAGGCGAATT \\
\hline $7[609]$ & $7[631]$ & ATTTGGCAAATCAACAGTTGAAA \\
\hline 4 4648] & $7[650]$ & GCATCGAGCCAGATATCTTTAGGACCTGAGGAAGGTTATC \\
\hline $7[651]$ & $7[673]$ & TAAGTAGAAGAACTCAAACTATCG \\
\hline $7[128]$ & $8[112]$ & TTCCGAATTGTAAACGTGTCGCCAGCATCGGTGCGGGCCT \\
\hline $8[153]$ & $8[131]$ & TCAGGCTGCGCAACTGTTGGGAA \\
\hline $7[170]$ & $8[154]$ & TTTTTATCCAATAAATCTCTACCCCGGTAAAACTAGCATG \\
\hline $8[195]$ & $8[173]$ & TTAACAAGAGAATCGATGAACGG \\
\hline $7[212]$ & $8[196]$ & TTTCACGAGAATGACCATTTTCATTTGGTCAATAACCTGT \\
\hline $8[237]$ & $8[215]$ & GCTTGACCATTAGATACATTTCG \\
\hline $7[254]$ & $8[238]$ & TTACCAATAAGGCTTGCAGTGCGGAAGTTTAGACTGGATA \\
\hline 8 [279] & $8[257]$ & ATTTTGCCAGAGGGGGTAATAGT \\
\hline $7[296]$ & $8[280]$ & $\begin{array}{l}\text { AGACGTCGTCACCCTCAGATCTTGACGCTGGCTGACCTTC } \\
\end{array}$ \\
\hline $8[321]$ & $8[299]$ & CCGAACGGTGTACAGACCAGGCG \\
\hline $7[338]$ & $8[322]$ & GGAGCAGCCACCACCCTTCGCATAACGACAATGACAACAA \\
\hline $8[363]$ & $8[341]$ & TGAACAGCTTGATACCGATAGTT \\
\hline $7[380]$ & $8[364]$ & TCAAGCAGAACCACCACTCACTCAGGTAGCCCGGAATAGG \\
\hline 8 [405] & $8[383]$ & GGTGCCGTCGAGAGGGTTGATAT \\
\hline 7[422] & $8[406]$ & AGCGCCACCACGGAATACGCCTCAGACCAGAGCCACCACC \\
\hline $8[447]$ & $8[425]$ & ATTCTTTTCATAATCAAAATCAC \\
\hline 7[464] & $8[448]$ & AAGCACAGAGCCTAATTATTGTTAGCGATTAAGACTCCTT \\
\hline 8[489] & 8 8[467] & AAAACGGAATACCCAAAAGAACT \\
\hline $7[506]$ & $8[490]$ & AAATCAGCCAGTAATAACACTATTTTTGAAGCCTTAAATC \\
\hline $8[531]$ & $8[509]$ & AACGAACCTCCCGACTTGCGGGA \\
\hline $7[548]$ & $8[532]$ & $\begin{array}{l}\text { TAAGATCTGTAAATCGTTGTTAATTGTAAAGCCAACGCTC } \\
\end{array}$ \\
\hline
\end{tabular}




\begin{tabular}{|c|c|c|}
\hline $8[573]$ & $8[551]$ & AAATGCGTTATACAAATTCTTAC \\
\hline $7[590]$ & $8[574]$ & AATCGTTGAGTAACATTGGAATTACCTAATTACATTTAAC \\
\hline $7[674]$ & $8[658]$ & GCCTTACGCTGCGCGTAAAATTATTTTTTGACGCTCAATC \\
\hline $8[46]$ & $9[62]$ & CAGCATCAACCGCACGGCGGGCCGTT \\
\hline $8[88]$ & $9[104]$ & AGCCTCCCCAGGGTCCGGCAAACGCG \\
\hline $8[130]$ & $9[146]$ & GGGCGTGAAATATTAGCGCCATTCGC \\
\hline $8[172]$ & $9[188]$ & TAATCGTAGCATTACCTGAGAGTCTG \\
\hline $8[214]$ & $9[230]$ & CAAATGGTTCAGAAGAACGAGTAGAT \\
\hline $8[256]$ & 9 [272] & AAAATTCCATTCAGGCTTTTGCAAAA \\
\hline $8[298]$ & $9[314]$ & CATAGAATTTGCGGTTTGAAAGAGGA \\
\hline $8[340]$ & $9[356]$ & GCGCCCGCACCCTCTCGAGGTGAATT \\
\hline $8[382]$ & $9[398]$ & AAGTAAGAGCCGCCAGTACCAGGCGG \\
\hline $8[424]$ & $9[440]$ & CGGAAGCACGCAAACTTATTAGCGTT \\
\hline $8[466]$ & $9[482]$ & GGCATAAGCGTCTTCGAGGAAACGCA \\
\hline $8[508]$ & $9[524]$ & GGTTTGCGCATTTTAACGCGAGGCGT \\
\hline $8[550]$ & $9[566]$ & CAGTAAGAACCTTGAGCCTGTTTAGT \\
\hline 8[592] & $9[608]$ & AAAATTTTTTAAAATGAGCAAAAGAA \\
\hline $8[634]$ & $9[650]$ & TACATAAATTCTGGGCACTAACAACT \\
\hline $3[79]$ & $10[80]$ & GTGGAACGACGGGCTCTCAACTT \\
\hline $3[121]$ & $10[122]$ & AATCAGTTAAAACGTGGGAGAAA \\
\hline $3[163]$ & $10[164]$ & TAAAGAGGCAAAATATTTTATAA \\
\hline $3[205]$ & $10[206]$ & GTCAGAATCAGGCAGGATTCGCG \\
\hline $3[247]$ & $10[248]$ & CTTGAAAACACCCTAACGGCATA \\
\hline $3[289]$ & $10[290]$ & GCTACGACAGCAACTAAAAACCG \\
\hline $3[331]$ & $10[332]$ & TACCGGGATAGCAATGAATATAT \\
\hline $3[373]$ & $10[374]$ & GGTCACGCCAGCACAGGAGTTAG \\
\hline $3[415]$ & $10[416]$ & GTTTATGTCACATGGGAATCCAC \\
\hline $3[457]$ & $10[458]$ & CAATCCAAAATACTGAACAGTAG \\
\hline 3[499] & $10[500]$ & TGTCCAAGTACCAGAAACCCCAG \\
\hline $3[541]$ & $10[542]$ & CATAGTTAATTTGTAAATGTCGC \\
\hline $3[583]$ & $10[584]$ & GGAATCGGAACATTGCACGTTAA \\
\hline $3[625]$ & $10[626]$ & AGACAACCTGAACAGTATTCGAC \\
\hline 11[681] & $10[668]$ & AAAGGGCGCTGGCAAGTATTGGC \\
\hline $0[53]$ & $11[62]$ & CGGTAGTACTCAATCCGCTGCTGGTCATGGTC \\
\hline 10 [79] & $11[92]$ & GTATGTGAAATTGTTATCC \\
\hline $0[95]$ & $11[104]$ & CCGGAAGACGTACAGCGCCGCGATTACAATTCC \\
\hline $10[121]$ & $11[134]$ & GGGCCGGAAGCATAAAGTG \\
\hline $0[137]$ & $11[146]$ & CATCAGCGTCTGGCCTTCCACAGGAACCTGGGG \\
\hline $10[163]$ & $11[176]$ & TCAGCTAACTCACATTAAT \\
\hline $0[179]$ & $11[188]$ & GCCTTATACCCTGTAATACCAATTCTTGCGCTC \\
\hline
\end{tabular}




\begin{tabular}{|c|c|c|c|}
\hline $10[205]$ & $11[218]$ & AGCAGTCGGGAAACCTGTC & \\
\hline $0[221]$ & $11[230]$ & CGAGCACAGACTTCAAATACCTCAAAAGCTGCA & \\
\hline $10[247]$ & $11[260]$ & AATAACGCGCGGGGAGAGG & \\
\hline $0[263]$ & $11[272]$ & CAGTCTTGATTTTAAGAACTCAACGTTGCGTAT & \\
\hline $10[289]$ & $11[302]$ & GATGTTTTTCTTTTCACCA & \\
\hline $0[305]$ & $11[314]$ & ACTACCTTTAAACGGGTAACAGGGAGACGGGCA & \\
\hline $10[331]$ & $11[344]$ & TCGTTCACCGCCTGGCCCT & \\
\hline $0[347]$ & $11[363]$ & AGCGTATCATTCCACAGACCCGCCACAGTTGCAGCAAGCG & \\
\hline $10[373]$ & $11[386]$ & TACCTGGTTTGCCCCAGCA & \\
\hline $0[389]$ & $11[398]$ & GAATTGTAGCCAGAATGGATCAGAGCAAATCCT & \\
\hline $10[415]$ & $11[428]$ & CCTCCGAAATCGGCAAAAT & \\
\hline $0[431]$ & 11[440] & ATTCAAGGGGAAGGTAAATGTGGCAAATAAATC & \\
\hline $10[457]$ & $11[470]$ & AAAAGATAGGGTTGAGTGT & \\
\hline 0 [473] & $11[482]$ & AAAAAAGGCAGCCTTTACAATCTTACCAGTTTG & \\
\hline $10[499]$ & $11[512]$ & CTATATTAAAGAACGTGGA & \\
\hline $0[515]$ & $11[524]$ & CTGAAAACCTGTTTATCAAACATGTAACGTCAA & \\
\hline $10[541]$ & 11[554] & CATTCTATCAGGGCGATGG & \\
\hline $0[557]$ & $11[566]$ & TACCTAATATCAAAATCATTCAATATTACGTGA & \\
\hline $10[583]$ & $11[596]$ & TGGCAAGTTTTTTGGGGTC & \\
\hline $0[599]$ & $11[608]$ & TTCTGGAATAATCCTGATTTTGCCCGGCCGTAA & \\
\hline $10[625]$ & $11[638]$ & AACACCCTAAAGGGAGCCC & \\
\hline $0[641]$ & $11[650]$ & CCGAACCCCCTAAAACATCGACCAGTTTAGAGC & \\
\hline $10[667]$ & $11[680]$ & AGACGGCGAACGTGGCGAG & \\
\hline $8[69]$ & $8[47]$ & TCGGTCATACCGGGGGTTTCTGC & Donor \\
\hline $8[111]$ & $8[89]$ & CTTTTTTTCGTCTCGTCGCTGGC & Donor \\
\hline $8[615]$ & 8 [593] & GTTGAAACAAACATCAAGAAAAC & Donor \\
\hline $8[657]$ & $8[635]$ & GTATTAGAGCCGTCAATAGATAA & Donor \\
\hline $7[86]$ & $8[70]$ & ATGAATCCCAGTCACGATCGAACGTGCCGGCCAGAGCACA & Acceptor \\
\hline $7[632]$ & $8[616]$ & GGAATAACAGAGATAGACATACAAACTTGAGGATTTAGAA & Acceptor \\
\hline $4[83]$ & $4[61]$ & GTACATCGACATCGTTAACGGCA & Biotin \\
\hline $4[251]$ & $4[229]$ & AACGCCAAAAGGCGGATGGCTTA & Biotin \\
\hline $4[461]$ & $4[439]$ & AAGAAACAATGACCGGAAACGTC & Biotin \\
\hline 5[665] & $4[672]$ & ATACCACCATCAGTGAGGCCAAACCGTTGTAGCAA & Biotin \\
\hline
\end{tabular}

\section{References}

(1) Schönle, A. Imspector Image Acquisition \& Analysis Software, V0.10

hhttp://www.imspector.de.

(2) Raab, M.; Vietz, C.; Stefani, F. D.; Acuna, G. P.; Tinnefeld, P. Shifting Molecular Localization 
by Plasmonic Coupling in a Single-Molecule Mirage. Nat. Commun. 2017, 8, 1-6. https://doi.org/10.1038/ncomms13966.

(3) ATTO-TEC Gmbh. Fluorescent Labels and Dyes Catalogue 2019/2020. 2019.

(4) Derr, N. D.; Goodman, B. S.; Jungmann, R.; Leschziner, A. E.; Shih, W. M.; Reck-Peterson, S. L. Tug-of-War in Motor Protein Ensembles Revealed with a Programmable DNA Origami Scaffold. Science 2012, 338 (6107), 662-665. https://doi.org/10.1126/science.1226734.

(5) Descloux, A.; Grußmayer, K. S.; Radenovic, A. Parameter-Free Image Resolution Estimation Based on Decorrelation Analysis. Nat. Methods 2019, 16 (9), 918-924. https://doi.org/10.1038/s41592-019-0515-7.

(6) Krull, A.; Buchholz, T.-O.; Jug, F. Noise2Void - Learning Denoising From Single Noisy Images. In 2019 IEEE/CVF Conference on Computer Vision and Pattern Recognition (CVPR); IEEE, 2019; Vol. 2019-June, pp 2124-2132. https://doi.org/10.1109/CVPR.2019.00223.

(7) van Rheenen, J.; Langeslag, M.; Jalink, K. Correcting Confocal Acquisition to Optimize Imaging of Fluorescence Resonance Energy Transfer by Sensitized Emission. Biophys. J. 2004, 86 (4), 2517-2529. https://doi.org/10.1016/S0006-3495(04)74307-6. 